\title{
Forecasting Multiple Time Series with One-Sided Dynamic Principal Components
}

\author{
Daniel Peña* \\ Department of Statistics and Institute of Financial Big Data \\ Universidad Carlos III de Madrid, Spain \\ and \\ Ezequiel Smucler \\ Instituto de Calculo \\ School of Exact and Natural Sciences \\ Universidad de Buenos Aires - CONICET, Argentina \\ and \\ Victor J. Yohai \\ Instituto de Calculo and Department of Mathematics \\ School of Exact and Natural Sciences \\ Universidad de Buenos Aires - CONICET, Argentina
}

\begin{abstract}
*Daniel Peña is Professor, Department of Statistics and Institute of Financial Big Data, Universidad Carlos III de Madrid, Calle Madrid 126, 28903 Getafe, España, (e-mail: daniel.pena@uc3m.es). Ezequiel Smucler is currently Postdoctoral Research Fellow, Department of Statistics, University of British Columbia, 3182 Earth Sciences Building, 2207 Main Mall Vancouver, BC, Canada V6T 1Z4 (e-mail: esmucler@cs.ubc.ca). Victor J. Yohai is Professor Emeritus, Mathematics Department, Faculty of Exact Sciences, Ciudad Universitaria, 1428 Buenos Aires, Argentina (e-mail: victoryohai@gmail.com). D.P. has been supported by Grant ECO2015-66593-P of MINECO/FEDER/UE. E.S. was partially funded by a CONICET Ph.D fellowship and by grant PIP 112-201101-00339 from CONICET.
\end{abstract}


We define one-sided dynamic principal components (ODPC) for time series as linear combinations of the present and past values of the series that minimize the reconstruction mean squared error. Previous definitions of dynamic principal components depend on past and future values of the series. For this reason, they are not appropriate for forecasting purposes. On the contrary, it is shown that the ODPC introduced in this paper can be successfully used for forecasting high-dimensional multiple time series. An alternating least squares algorithm to compute the proposed ODPC is presented. We prove that for stationary and ergodic time series the estimated values converge to their population analogues. We also prove that asymptotically, when both the number of series and the sample size go to infinity, if the data follows a dynamic factor model, the reconstruction obtained with ODPC converges, in mean squared error, to the common part of the factor model. Monte Carlo results shows that forecasts obtained by the ODPC compare favourably with other forecasting methods based on dynamic factor models.

Keywords: dimensionality reduction; high-dimensional time series; dynamic factor models 


\section{Introduction}

Forecasting a large number of cross-correlated time series is a difficult problem. Building a multivariate VARMA model is only possible when the number of series is small compared to the sample size. Therefore, other alternatives have been explored. Box and Tiao (1977) introduced linear combinations of the series with maximum predictability. Litterman (1986) proposed Bayesian VAR models with shrinking prior distributions to control the number of parameters. Ahn and Reinsel (1988) addressed this problem by introducing reduced-rank autoregressive models. Tiao and Tsay (1989) presented ways to simplify the construction of VARMA models by identifying scalar components. However, the currently most popular procedures for large data sets are based on dynamic factor models, where the relationship between the series and the factor can be contemporaneous, or with lags. Stock and Watson (2002) use the contemporaneous model for forecasting assuming that all the variables follow the same dynamic factor model. Then, the forecast of a given variable can be written as the sum of the forecast of the common component, driven by the factors, plus the univariate forecasts of the idiosyncratic component. They used principal components of the explanatory variables to obtain consistent estimators of the factor effects and fitted univariate autoregressive models to forecast the idiosyncratic component. Their method showed a good performance in simulated and real macroeconomic data. This work also explains why univariate forecasts are improved by using as a regressor a weighted average of all the series. This procedure, called forecast pooling (see Garcia-Ferrer et al. (1987)),

can be justified by assuming a common factor in the series, as shown by Peña and Poncela (2004).

Forni et al. (2000) proposed a general dynamic factor model assuming lagged relationships between the series and the factors. They allow for an infinite number of factor lags 
and low correlation between any two idiosyncratic components. Forni et al. (2005) proposed a one-sided method of estimation of the common part of a dynamic factor model for forecasting. The forecasts generated with this procedure have been compared tothe ones derived by Stock and Watson (2002) and the results are mixed (see Forni et al. (2015a)). A modified forecasting approach was proposed by Forni et al. (2015b), although again, as shown in Forni et al. (2015b), the results are mixed.

Peña and Yohai (2016), following Brillinger's idea of dynamic principal components, Brillinger (1964, 1981), proposed components that provide an optimal reconstruction of the series in finite samples, but dropping Brillingers's assumption that the components are linear combinations of the data. However, this approach is not expected to work well in forecasting problems as the last values of the dynamic principal components have been computed with smaller number of observations than the central values.

In Section 2 of this paper we define one-sided dynamic principal components (ODPC) as linear combinations of present and previous values of the series which have optimal reconstruction performance, that is, they minimize a mean squared error reconstruction criterion. Following Hotelling's original spirit of principal components, our definition is not based on assuming any model for the vector time series. We show how to forecast future values of the series using the proposed ODPC and a univariate forecasting method. We present two properties of the proposed estimator. In Section 3 we prove that for stationary and ergodic time series the estimated values converge to their population analogues. We also prove, in Section 4, that asymptotically, when both the number of series and the sample size goes to infinity, if the data follows a dynamic factor model, the reconstruction obtained with ODPC converges, in mean squared error, to the common part of the factor model. In Sections 5 and 6 we illustrate with Monte Carlo simulations and with a real data example that our forecasting procedure compares favourably with other forecasting methods based 
on dynamic factor models. We discuss possible strategies for choosing the number of components and lags used to define them in Section 7 . Finally, some conclusions and possible extensions are discussed in Section 8. Section 9 is a technical appendix containing the proofs of our main theoretical results.

\section{One Sided Dynamic Components and their compu- tation}

Consider the vector time series $\mathbf{z}_{1}, \ldots, \mathbf{z}_{T}$, where $\mathbf{z}_{t}=\left(z_{t, 1}, \ldots, z_{t, m}\right)^{\prime}$. Let $\mathbf{Z}$ be the data matrix of dimension $T \times m$ where each row is $\mathbf{z}_{t}^{\prime}$. Consider integer numbers $k_{1}^{1}, k_{2}^{1} \geq 0$. Let $\mathbf{a}=\left(\mathbf{a}_{0}^{\prime}, \ldots, \mathbf{a}_{k_{1}^{1}}^{\prime}\right)^{\prime}$, where $\mathbf{a}_{h}^{\prime}=\left(a_{h, 1}, \ldots, a_{h, m}\right)$, be a vector of dimension $m\left(k_{1}+1\right) \times 1$, let $\boldsymbol{\alpha}^{\prime}=\left(\alpha_{1}, \ldots, \alpha_{m}\right)$ and $\mathbf{B}$ the matrix that has coefficients $b_{h, j}$ and dimension $\left(k_{2}^{1}+1\right) \times m$. We can define the first one-sided dynamic principal component with $k_{1}^{1}$ lags as the vector

$$
f_{t}=\sum_{j=1}^{m} \sum_{h=0}^{k_{1}^{1}} a_{h, j} z_{t-h, j} \quad t=k_{1}^{1}+1, \ldots, T
$$

and use this component to reconstruct the series using $k_{2}^{1}$ lags of the component as

$$
z_{t, j}^{R}(\mathbf{a}, \boldsymbol{\alpha}, \mathbf{B})=\alpha_{j}+\sum_{h=0}^{k_{2}^{1}} b_{h, j} f_{t-h} .
$$

The values $\left(k_{1}^{1}, k_{2}^{1}\right)$ that define the first dynamic principal components will be discussed later. Suppose now they are given. Then, the theoretical optimal values of $\mathbf{a}, \boldsymbol{\alpha}$ and $\mathbf{B}$ can be defined as those that minimize the mean squared error in the reconstruction of the data, that is, calling $\mathbb{E}$ the expectation operator, as the solutions of

$$
\left(\mathbf{a}^{*}, \boldsymbol{\alpha}^{*}, \mathbf{B}^{*}\right)=\arg \min _{\mathbf{a}, \boldsymbol{\alpha}, \mathbf{B}} \frac{1}{T-\left(k_{1}^{1}+k_{2}^{2}\right)} \sum_{j=1}^{m} \sum_{t=\left(k_{1}^{1}+k_{2}^{2}\right)+1}^{T} \mathbb{E}\left(z_{t, j}-z_{t, j}^{R}(\mathbf{a}, \boldsymbol{\alpha}, \mathbf{B})\right)^{2}
$$


Natural estimators of $\left(\mathbf{a}^{*}, \boldsymbol{\alpha}^{*}, \mathbf{B}^{*}\right)$ can be defined as solutions of

$$
\arg \min _{\mathbf{a}, \boldsymbol{\alpha}, \mathbf{B}} \operatorname{MSE}(\mathbf{a}, \boldsymbol{\alpha}, \mathbf{B})
$$

where

$$
\operatorname{MSE}(\mathbf{a}, \boldsymbol{\alpha}, \mathbf{B})=\frac{1}{T-\left(k_{1}^{1}+k_{2}^{1}\right)} \sum_{j=1}^{m} \sum_{t=\left(k_{1}^{1}+k_{2}^{1}\right)+1}^{T}\left(z_{t, j}-z_{t, j}^{R}(\mathbf{a}, \boldsymbol{\alpha}, \mathbf{B})\right)^{2}
$$

Note that if $(\mathbf{a}, \boldsymbol{\alpha}, \mathbf{B})$ is a solution of (2) then $(\gamma \mathbf{a}, \boldsymbol{\alpha}, \mathbf{B} / \gamma)$ will be one as well. Hence, we define the optimal $\widehat{\mathbf{a}}, \widehat{\boldsymbol{\alpha}}$ and $\widehat{\mathbf{B}}$ as any solution of

$$
\operatorname{MSE}(\widehat{\mathbf{a}}, \widehat{\boldsymbol{\alpha}}, \widehat{\mathbf{B}})=\min _{\|\mathbf{a}\|=1, \boldsymbol{\alpha}, \mathbf{B}} \operatorname{MSE}(\mathbf{a}, \boldsymbol{\alpha}, \mathbf{B}) .
$$

Conditions to guarantee the existence of at least one solution of (4) will be given in Section 3.

Let

$$
\widehat{f}_{t}=\sum_{j=1}^{m} \sum_{h=0}^{k_{1}^{1}} \widehat{a}_{h, j} z_{t-h, j}
$$

and $\widehat{z}_{t, j}=z_{t, j}^{R}(\widehat{\mathbf{a}}, \widehat{\boldsymbol{\alpha}}, \widehat{\mathbf{B}})=\widehat{\alpha}_{j}+\sum_{h=0}^{k_{2}^{1}} \widehat{b}_{h, j} \widehat{f}_{t-h}$ be the corresponding optimal reconstruction of the $j$-th series at period $t, 1 \leq j \leq m,\left(k_{1}^{1}+k_{2}^{1}\right)+1 \leq t \leq T$. We define the second one-sided dynamic principal component with $\left(k_{1}^{2}, k_{2}^{2}\right)$ lags as the first one-sided dynamic principal component of the residuals $z_{t, j}-\widehat{z}_{t, j}, 1 \leq j \leq m,\left(k_{1}^{1}+k_{2}^{1}\right)+1 \leq t \leq T$. Higher order principal components are defined similarly. Note that if we compute $q$ one-sided principal components, each with $\left(k_{1}^{i}, k_{2}^{i}\right)$ lags, $1 \leq i \leq q$, we will only be able to reconstruct the periods $\sum_{i=1}^{q}\left(k_{1}^{i}+k_{2}^{i}\right)+1, \ldots, T$. The superscript $i$, indicating the principal component in the vector of lags $\left(k_{1}^{i}, k_{2}^{i}\right)$ will generally be omitted when no confusion could arise.

In order to derive an algorithm to compute estimators of $\mathbf{a}, \boldsymbol{\alpha}$ and $\mathbf{B}$, we need first to express the objective function, $\operatorname{MSE}(\mathbf{a}, \boldsymbol{\alpha}, \mathbf{B})$, in a more manageable form. To this end, we will introduce further notation. Throughout this paper $\|\cdot\|$ will stand for the Euclidean 
norm for vectors and the spectral norm for matrices, whereas $\|\cdot\|_{F}$ will stand for the Frobenius norm for matrices. $\mathbf{A}^{\dagger}$ will stand for the Moore-Penrose pseudo-inverse of a matrix A. For $h=0, \ldots,\left(k_{1}+k_{2}\right)$, let $\mathbf{Z}_{h}$ be the $\left(T-\left(k_{1}+k_{2}\right)\right) \times m$ data matrix

$$
\mathbf{Z}_{h}=\left(\begin{array}{c}
\mathbf{z}_{h+1}^{\prime} \\
\mathbf{z}_{h+2}^{\prime} \\
\mathbf{z}_{T-\left(k_{1}+k_{2}\right)+h}^{\prime}
\end{array}\right)
$$

For $l=k_{1}, \ldots,\left(k_{1}+k_{2}\right)$, let $\mathbf{Z}_{l, 0}=\left[\mathbf{Z}_{l}, \mathbf{Z}_{l-1}, \ldots, \mathbf{Z}_{l-k_{1}}\right]$ be a $\left(T-\left(k_{1}+k_{2}\right)\right) \times m\left(k_{1}+1\right)$ matrix. Then, letting $\mathbf{g}_{l}=\left(f_{l+1}, \ldots, f_{T-\left(k_{1}+k_{2}\right)+l}\right)^{\prime}$ be a vector of dimension $\left(T-\left(k_{1}+k_{2}\right)\right) \times 1$ we have $\mathbf{g}_{l}=\mathbf{Z}_{l, 0}$ a. Let $\mathbf{D}$ be the matrix of dimension $\left(k_{2}+2\right) \times m$ given by $\mathbf{D}=\left(\begin{array}{l}\boldsymbol{\alpha}^{\prime} \\ \mathbf{B}\end{array}\right)$.

The reconstruction of the values of the $\mathbf{Z}_{k_{1}+k_{2}}$ matrix using $\mathbf{a}, \boldsymbol{\alpha}$ and $\mathbf{B}$ can be written as a matrix $\widehat{\mathbf{Z}}_{k_{1}+k_{2}}$ of the same dimension, $\left(T-\left(k_{1}+k_{2}\right)\right) \times m$, as

$$
\widehat{\mathbf{Z}}_{k_{1}+k_{2}}=\mathbf{F}_{k_{1}, k_{2}} \mathbf{D}
$$

where $\mathbf{F}_{k_{1}, k_{2}}=\left[\mathbf{1}_{T-\left(k_{1}+k_{2}\right)}, \mathbf{g}_{\left(k_{1}+k_{2}\right)}, \mathbf{g}_{\left(k_{1}+k_{2}\right)-1}, \ldots, \mathbf{g}_{k_{1}}\right]$ is a matrix with dimensions $(T-$ $\left.\left(k_{1}+k_{2}\right)\right) \times\left(k_{2}+2\right)$ and $\mathbf{1}_{T-\left(k_{1}+k_{2}\right)}$ is a vector of length $T-\left(k_{1}+k_{2}\right)$ with all its coordinates equal to one. Note that $\mathbf{F}_{k_{1}, k_{2}}=\mathbf{F}_{k_{1}, k_{2}}(\mathbf{a})$, even though this dependence will not in general be made explicit in the notation.

Let $\mathbf{C}$ be the matrix with dimensions $\left(T-\left(k_{1}+k_{2}\right)\right)\left(k_{2}+1\right) \times m\left(k_{1}+1\right)$ given by

$$
\mathbf{C}=\left(\begin{array}{c}
\mathbf{Z}_{k_{1}+k_{2}, 0} \\
\vdots \\
\mathbf{Z}_{k_{1}, 0}
\end{array}\right)
$$

Note that

$$
\mathbf{F}_{k_{1}, k_{2}}=\left(\mathbf{1}_{T-\left(k_{1}+k_{2}\right)}, \mathbf{Z}_{k_{1}+k_{2}, 0} \mathbf{a}, \ldots, \mathbf{Z}_{k_{1}, 0} \mathbf{a}\right)
$$


Hence

$$
\operatorname{vec}\left(\mathbf{F}_{k_{1}, k_{2}}\right)^{\prime}=\left(\mathbf{1}_{T-\left(k_{1}+k_{2}\right)}^{\prime},\left(\mathbf{Z}_{k_{1}+k_{2}, 0} \mathbf{a}\right)^{\prime}, \ldots,\left(\mathbf{Z}_{k_{1}, 0} \mathbf{a}\right)^{\prime}\right)=\left(\begin{array}{c}
\mathbf{1}_{T-\left(k_{1}+k_{2}\right)} \\
\mathbf{C a}
\end{array}\right)^{\prime}
$$

and

$$
\begin{aligned}
\operatorname{vec}\left(\widehat{\mathbf{Z}}_{k_{1}+k_{2}}\right)=\operatorname{vec}\left(\mathbf{F}_{k_{1}, k_{2}} \mathbf{D}\right) & =\left(\mathbf{D}^{\prime} \otimes \mathbf{I}_{T-\left(k_{1}+k_{2}\right)}\right) \operatorname{vec}\left(\mathbf{F}_{k_{1}, k_{2}}\right) \\
& =\left(\mathbf{D}^{\prime} \otimes \mathbf{I}_{T-\left(k_{1}+k_{2}\right)}\right)\left(\begin{array}{c}
\mathbf{1}_{T-\left(k_{1}+k_{2}\right)} \\
\mathbf{C a}
\end{array}\right) .
\end{aligned}
$$

Then, $(\widehat{\mathbf{a}}, \widehat{\boldsymbol{\alpha}}, \widehat{\mathbf{B}})$ can be obtained by minimizing

$$
\left\|\mathbf{Z}_{k_{1}+k_{2}}-\widehat{\mathbf{Z}}_{k_{1}+k_{2}}\right\|_{F}^{2}=\left\|\mathbf{Z}_{k_{1}+k_{2}}-\mathbf{F}_{k_{1}, k_{2}} \mathbf{D}\right\|_{F}^{2}=\left\|\operatorname{vec}\left(\mathbf{Z}_{k_{1}+k_{2}}\right)-\operatorname{vec}\left(\widehat{\mathbf{Z}}_{k_{1}+k_{2}}\right)\right\|^{2}
$$

subject to $\|\widehat{\mathbf{a}}\|=1$. Note that

$$
\begin{aligned}
& \left\|\operatorname{vec}\left(\mathbf{Z}_{k_{1}+k_{2}}\right)-\operatorname{vec}\left(\widehat{\mathbf{Z}}_{k_{1}+k_{2}}\right)\right\|^{2}= \\
& \left\|\operatorname{vec}\left(\mathbf{Z}_{k_{1}+k_{2}}\right)-\left(\mathbf{D}^{\prime} \otimes \mathbf{I}_{T-\left(k_{1}+k_{2}\right)}\right)\left(\begin{array}{c}
\mathbf{1}_{T-\left(k_{1}+k_{2}\right)} \\
\mathbf{C a}
\end{array}\right)\right\|^{2}= \\
& \left\|\operatorname{vec}\left(\mathbf{Z}_{k_{1}+k_{2}}\right)-\left(\boldsymbol{\alpha} \otimes \mathbf{I}_{T-\left(k_{1}+k_{2}\right)}\right) \mathbf{1}_{T-\left(k_{1}+k_{2}\right)}-\left(\mathbf{B}^{\prime} \otimes \mathbf{I}_{T-\left(k_{1}+k_{2}\right)}\right) \mathbf{C a}\right\|^{2}
\end{aligned}
$$

For a fixed $\mathbf{D}, \widehat{\mathbf{a}}$ can be computed by least squares

$$
\widehat{\mathbf{a}}=\left(\left(\mathbf{B}^{\prime} \otimes \mathbf{I}_{T-\left(k_{1}+k_{2}\right)}\right) \mathbf{C}\right)^{\dagger}\left(\operatorname{vec}\left(\mathbf{Z}_{k_{1}+k_{2}}\right)-\left(\boldsymbol{\alpha} \otimes \mathbf{I}_{T-\left(k_{1}+k_{2}\right)}\right) \mathbf{1}_{T-\left(k_{1}+k_{2}\right)}\right) .
$$

and then standardized to unit norm. On the other hand, for a fixed $\mathbf{F}_{k_{1}, k_{2}}$, the optimal D can also be computed by least squares

$$
\widehat{\mathbf{D}}=\left(\mathbf{F}_{k_{1}, k_{2}}\right)^{\dagger} \mathbf{Z}_{k_{1}+k_{2}}
$$

Then, $\widehat{\boldsymbol{\alpha}}$ is given by the first row of $\widehat{\mathbf{D}}$ and $\widehat{\mathbf{B}}$ is given by the last $k_{2}+1$ rows of $\widehat{\mathbf{D}}$. 


\subsection{Computing algorithm}

We propose the following alternating Least Squares algorithm for computing $\widehat{\mathbf{a}}, \widehat{\mathbf{D}}$. Let $\mathbf{a}^{(i)}, \mathbf{D}^{(i)}$ and $\mathbf{f}^{(i)}$ be the values of $\mathbf{a}, \mathbf{D}$ and $\mathbf{f}$ corresponding to the $i$-th iteration. Let $\delta \in(0,1)$, a tolerance parameter to stop the iterations. Write $\operatorname{MSE}(\mathbf{a}, \mathbf{D})=\operatorname{MSE}(\mathbf{a}, \boldsymbol{\alpha}, \mathbf{B})$. In order to define the algorithm it is enough to give an initial value of the component, $\mathbf{f}=\left(f_{k_{1}+1}, \ldots, f_{T}\right)^{\prime}$, say $\mathbf{f}^{(0)}$, and describe a rule to compute $\mathbf{D}^{(i+1)}, \mathbf{a}^{(i+1)}$ and $\mathbf{f}^{(i+1)}$ from $\mathbf{f}^{(i)}$. This can be done as follows:

1. Given $\mathbf{f}^{(i)}$ define $\mathbf{D}^{(i+1)}$ by (9), where $\mathbf{F}_{k_{1}, k_{2}}$ corresponds to $\mathbf{f}^{(i)}$.

2. Compute $\mathbf{a}_{*}^{(i+1)}$ by (일 with $\mathbf{D}=\mathbf{D}^{(i+1)}$ and let $\mathbf{a}^{(i+1)}=\mathbf{a}_{*}^{(i+1)} /\left\|\mathbf{a}_{*}^{(i+1)}\right\|$.

3. The $t$-th coordinate of $\mathbf{f}^{(i+1)}$ is given by (1) with $\mathbf{a}=\mathbf{a}^{(i+1)}$.

The stopping rule is as follows: Stop when

$$
\frac{\operatorname{MSE}\left(\mathbf{a}^{(i)}, \mathbf{D}^{(i)}\right)-\operatorname{MSE}\left(\mathbf{a}^{(i+1)}, \mathbf{D}^{(i+1)}\right)}{\operatorname{MSE}\left(\mathbf{a}^{(i)}, \mathbf{D}^{(i)}\right)} \leq \delta
$$

Clearly in this algorithm at each step the MSE decreases and therefore it converges to a local minimum. To obtain a global minimum the initial value $\mathbf{f}^{(0)}$ should be close enough to the optimal one. We propose to take $\mathbf{f}^{(0)}$ as the last $T-k_{1}$ coordinates of the first ordinary principal component of the data. Alternatively, the first Generalized Dynamic Principal Component proposed by Peña and Yohai (2016) could be used.

Note that since the matrix $\left(\mathbf{B}^{\prime} \otimes \mathbf{I}_{T-\left(k_{1}+k_{2}\right)}\right) \mathbf{C}$ has dimensions $m\left(T-\left(k_{1}+k_{2}\right)\right) \times m\left(k_{2}+1\right)$, solving the associated least squares problem can be time consuming for high-dimensional (large $m$ ) problems. The iterative nature of the algorithm we propose implies that this least squares problem will have to be solved several times for different $\mathbf{B}$ matrices. However, note that since the matrix $\mathbf{B}^{\prime} \otimes \mathbf{I}_{T-\left(k_{1}+k_{2}\right)}$ is sparse, it can be stored efficiently, and multiplying it with a vector is relatively fast. We found that for problems with a moderately large 
$m$, the following modification of our algorithm works generally faster: instead of finding the optimal $\mathbf{a}^{(i+1)}$ corresponding to $\mathbf{D}^{(i+1)}$, just do one iteration of coordinate descent for $\mathbf{a}^{(i+1)}$.

\section{$2.2 \quad$ Forecasting}

Suppose we have fitted $q$ dynamic principal components to the data, each with $\left(k_{1}^{i}, k_{2}^{i}\right)$ lags, $i=1, \ldots, q$. Let $\widehat{\mathbf{f}}_{T}^{i}$ be the vector with the estimated values for the $i$-th dynamic principal component and $\widehat{\mathbf{B}}^{i}, \widehat{\boldsymbol{\alpha}}^{i}$ be the corresponding loadings and intercepts. Suppose we have decided upon a procedure to forecast each of these dynamic principal components

separately, and let $\widehat{f}_{T+h \mid T}^{i}$ for $h>0$ be the forecast of $f_{T+h}^{i}$ with information until time $T$. We can obtain an $h$-steps ahead forecast of $\mathbf{z}_{T}$ as

$$
\widehat{z}_{T+h \mid T, j}=\sum_{i=1}^{q}\left(\widehat{\alpha}_{j}^{i}+\sum_{v=0}^{k_{2}^{i}} \widehat{b}_{v, j}^{i} \widehat{f}_{T+h-v \mid T}^{i}\right) \quad j=1, \ldots, m .
$$

\section{$3 \quad$ Wald type consistency for stationary data}

In this section we prove a consistency result for our procedure in the case of stationary and ergodic vector time series. First, in Proposition 10 we show that, asymptotically and with probability one, problem (4) is well defined. In Proposition 2 we show that the population version of (41), obtained replacing means by expectations is well defined. Finally, in Theorem 1. we prove that the distance between any given solution of (4) and the set of solutions of the population problem converges almost surely to zero.

We will assume that the process $\mathbf{z}_{t}, t \geq 1$, defined in a probability space $(\Omega, \mathcal{F}, \mathbb{P})$, is strictly stationary and ergodic. We note that if instead one assumes weak second order stationarity and ergodicity, similar results can be obtained, but with convergences in 
probability instead of almost surely. Let $\mathbf{z}$ denote a random variable with the common distribution of the $\mathbf{z}_{t}$ s. Given a square matrix $\mathbf{M}$, let $\lambda_{\min }(\mathbf{M})$ and $\lambda_{\max }(\mathbf{M})$ denote the smallest and largest (in absolute value) eigenvalues of $\mathbf{M}$ respectively. Moreover, $\operatorname{Tr}(\mathbf{M})$ will denote the trace of $\mathbf{M}$. We will assume that $\mathbb{E} \mathbf{z}=0$ and that $\mathbb{E}\|\mathbf{z}\|^{2}<\infty$. Let $\boldsymbol{\Sigma}(l)=\mathbb{E} \mathbf{z}_{t} \mathbf{z}_{t-l}^{\prime}$ be the lag $l$ autocovariance matrix of the process $\mathbf{z}_{t}$.

Note that (3) can be written as

$$
\operatorname{MSE}(\mathbf{a}, \boldsymbol{\alpha}, \mathbf{B})=\frac{1}{T-\left(k_{1}+k_{2}\right)} \sum_{t=\left(k_{1}+k_{2}\right)+1}^{T}\left\|\mathbf{z}_{t}-\widehat{\mathbf{z}}_{t}\right\|^{2},
$$

where $\widehat{\mathbf{z}}_{t}$ is the vector of length $m$ with coordinates

$$
\widehat{z}_{t, j}=\widehat{\alpha}_{j}+\sum_{h=0}^{k_{2}} \widehat{b}_{h, j} \widehat{f}_{t-h}, j=1, \ldots, m .
$$

Let $\mathbf{x}_{t}^{\prime}=\left(\mathbf{z}_{t}^{\prime}, \ldots, \mathbf{z}_{t-k_{1}}^{\prime}\right)$, so that given any $\mathbf{a}$ with $\|\mathbf{a}\|=1$, the corresponding component at time $t, f_{t}$, is given by $\mathbf{a}^{\prime} \mathbf{x}_{t}$. The lag $l$ autocovariance matrix of $\mathbf{x}_{t}$ is given by

$$
\mathbf{V}(l)=\left(\begin{array}{cccc}
\Sigma(l) & \Sigma(l+1) & \ldots & \boldsymbol{\Sigma}\left(l+k_{1}\right) \\
\vdots & \vdots & & \vdots \\
\Sigma\left(l-k_{1}\right) & \Sigma\left(l-k_{1}+1\right) & \ldots & \boldsymbol{\Sigma}(l)
\end{array}\right)
$$

Fix a with $\|\mathbf{a}\|=1$, then the covariance matrix of the vector

$$
\left(1, \mathbf{a}^{\prime} \mathbf{x}_{t}, \mathbf{a}^{\prime} \mathbf{x}_{t-1}, \ldots, \mathbf{a}^{\prime} \mathbf{x}_{t-k_{2}}\right)
$$

is

$$
\mathcal{S}(\mathbf{a})=\left(\begin{array}{ccccc}
1 & 0 & 0 & \ldots & 0 \\
0 & \mathbf{a}^{\prime} \mathbf{V}(0) \mathbf{a} & \mathbf{a}^{\prime} \mathbf{V}(1) \mathbf{a} & \ldots & \mathbf{a}^{\prime} \mathbf{V}\left(k_{2}\right) \mathbf{a} \\
\vdots & \vdots & \vdots & & \vdots \\
0 & \mathbf{a}^{\prime} \mathbf{V}\left(-k_{2}\right) \mathbf{a} & \mathbf{a}^{\prime} \mathbf{V}\left(-k_{2}+1\right) \mathbf{a} & \ldots & \mathbf{a}^{\prime} \mathbf{V}(0) \mathbf{a}
\end{array}\right) .
$$

We will need the following assumption. 
Condition 1. There exists $\eta<1$ such that

$$
\mathbb{P}\left(\sum_{h=0}^{k_{2}} v_{h}\left(\mathbf{a}^{\prime} \mathbf{x}_{t-h}\right)=v_{k_{2}+1}\right) \leq \eta
$$

for all $\mathbf{a} \in \mathbb{R}^{m\left(k_{1}+1\right)}$ with $\|\mathbf{a}\|=1$ and $\mathbf{v}=\left(v_{0}, \ldots, v_{k_{2}}, v_{k_{2}+1}\right)$ such that $\mathbf{v} \neq 0$. That is, for any $\mathbf{a}$, there is no deterministic linear relation between the values $f_{t}, \ldots, f_{t-k_{2}}$.

It follows from Condition 1 that $\inf _{\|\mathbf{a}\|=1} \lambda_{\min }(\mathcal{S}(\mathbf{a}))>0$. Note that $\sup _{\|\mathbf{a}\|=1} \lambda_{\max }(\mathcal{S}(\mathbf{a}))<$ $\infty$ always holds.

Proposition 1 shows that, asymptotically and with probability one, there exists at least one solution of (4). Write $\operatorname{MSE}(\mathbf{a}, \mathbf{D})$ for $\operatorname{MSE}(\mathbf{a}, \boldsymbol{\alpha}, \mathbf{B})$, where $\mathbf{D}=\left(\begin{array}{l}\boldsymbol{\alpha}^{\prime} \\ \mathbf{B}\end{array}\right)$.

Proposition 1. Assume Condition 1 holds. Then, with probability one, there exists $T_{0}$ such that for all $T>T_{0}$, $\arg \min _{\|\mathbf{a}\|=1, \mathbf{D}} M S E(\mathbf{a}, \mathbf{D})$ has at least one solution.

Let

$$
\operatorname{MSE}_{0}(\mathbf{a}, \boldsymbol{\alpha}, \mathbf{B})=\mathbb{E}\left\|\mathbf{z}_{t}-\mathbf{z}_{t}^{R}(\mathbf{a}, \boldsymbol{\alpha}, \mathbf{B})\right\|^{2}
$$

be the population version of (3). It is easy to verify that $\mathrm{MSE}_{0}$ is continuous. Let

$$
\mathcal{I}=\left\{\left(\mathbf{a}^{*}, \mathbf{D}^{*}\right): \operatorname{MSE}_{0}\left(\mathbf{a}^{*}, \mathbf{D}^{*}\right)=\inf _{\|\mathbf{a}\|=1, \mathbf{D}} \operatorname{MSE}_{0}(\mathbf{a}, \mathbf{D})\right\} .
$$

Proposition 2 entails that $\mathcal{I}$ is non-empty.

Proposition 2. Assume Condition 1 holds. Then $\inf _{\|\mathbf{a}\|=1} M S E_{0}(\mathbf{a}, \mathbf{D}) \rightarrow+\infty$ when $\|\mathbf{D}\|_{F} \rightarrow+\infty$.

Let $d((\mathbf{a}, \mathbf{D}), \mathcal{I})=\inf \left\{\left\|\mathbf{a}-\mathbf{a}^{*}\right\|+\left\|\mathbf{D}-\mathbf{D}^{*}\right\|_{F}:\left(\mathbf{a}^{*}, \mathbf{D}^{*}\right) \in \mathcal{I}\right\}$.

Theorem 1. [Consistency] Assume Condition 1 holds. Let $(\widehat{\mathbf{a}}, \widehat{\boldsymbol{\alpha}}, \widehat{\mathbf{B}})$ be a solution of (4) and $\widehat{\mathbf{D}}^{\prime}=\left(\begin{array}{ll}\widehat{\boldsymbol{\alpha}} & \widehat{\mathbf{B}}^{\prime}\end{array}\right)$. Then $d((\widehat{\mathbf{a}}, \widehat{\mathbf{D}}), \mathcal{I}) \stackrel{\text { a.s. }}{\rightarrow} 0$. 


\section{Consistency in the dynamic factor model}

In this section we deal with the interesting case where the series follow a stationary dynamic factor model. In Theorem 3 we prove a consistency result for this situation: asymptotically, when both the number of series and the sample size go to infinity, the reconstruction obtained with ODPC converges in mean-square to the common part of the factor model.

Suppose we have observations, $\mathbf{z}_{1}, \ldots, \mathbf{z}_{T}, \mathbf{z}_{t}^{\prime}=\left(z_{t, 1}, \ldots, z_{t, m}\right)$, of a double indexed stochastic process $\left\{z_{t, j}: t \in \mathbb{Z}, j \in \mathbb{N}\right\}$. Consider the following dynamic factor model with one factor, say $f_{t}$, and a finite dimensional factor space. That is,

$$
z_{t, j}=\sum_{h=0}^{k_{2}} b_{h, j} f_{t-h}+e_{t, j}, \quad t=1, \ldots, T, \quad j=1, \ldots, m,
$$

where the $e_{t, j}$ for $j=1, \ldots, m$ and $f_{t}$ are stationary processes and $b_{h, j}$ the factor loadings. This can be expressed in the form of a factor model, with $k_{2}+1$ static factors, as

$$
\mathbf{z}_{t}=\mathbf{B}^{\prime} \mathbf{f}_{t}+\mathbf{e}_{t}, \quad t=1, \ldots, T
$$

where $\mathbf{e}_{t}=\left(e_{t, 1}, \ldots, e_{t, m}\right)^{\prime}, \mathbf{B} \in \mathbb{R}^{\left(k_{2}+1\right) \times m}$ is the matrix with entries $b_{h, j}$ and $\mathbf{f}_{t}=\left(f_{t}, \ldots, f_{t-k_{2}}\right)^{\prime}$. For $h=0, \ldots, k_{2}$ let $\mathbf{b}_{h}=\left(b_{h, 1}, \ldots, b_{h, m}\right)^{\prime}$. The term $\boldsymbol{\chi}_{t}=\mathbf{B}^{\prime} \mathbf{f}_{t}$ is usually called the common part of the model. We will need the following assumptions.

\section{Condition 2.}

(a) $\mathbf{B B}^{\prime} / m \rightarrow \mathbf{I}_{k_{2}+1}$.

(b) $\mathbf{e}_{t}$ and $f_{t}$ are second order stationary, $\mathbb{E} \mathbf{e}_{t}=\mathbf{0}_{m}$ and $\mathbb{E} f_{t}=0$. Let $\boldsymbol{\Sigma}^{e}(l)$ be the lag $l$ autocovariance matrix of $\mathbf{e}_{t}$. Then $\lambda_{\max }\left(\boldsymbol{\Sigma}^{e}(0)\right)=O(1)$.

(c) $\mathbf{e}_{t}$ is uncorrelated with $\mathbf{f}_{t}$ at all leads and lags. 
Condition 2(a) is a standardization assumption. It appears, for example, in Peña and Box (1987). See also Bai and Ng (2002). Conditions 2(b) and (c) allow for weak cross-sectional correlations in the idiosyncratic part.

The following theorem shows that the population reconstruction mean squared error of the ODPC procedure is essentially bounded by the mean variance of the idiosyncratic part. This can also be interpreted as a sequential limit asymptotic: first let $T$ go to infinity for fixed $m$ and then let $m$ go to infinity. See Connor and Korajczyk (1993) for another example of sequential limit asymptotics. To keep the notation light, the theorem is stated and proved for the case in which an intercept $\boldsymbol{\alpha}$ is not included in the definition of the ODPC; the adjustments to include $\boldsymbol{\alpha}$ are straightforward.

Theorem 2. Assume Conditions 1 and 2 hold. Then as $m \rightarrow \infty$

$$
\frac{1}{m} M S E_{0}\left(\mathbf{a}^{*}, \mathbf{B}^{*}\right) \leq \frac{1}{m} \sum_{j=1}^{m} \mathbb{E} e_{t, j}^{2}+o(1), \quad \text { for all }\left(\mathbf{a}^{*}, \mathbf{B}^{*}\right) \in \mathcal{I} .
$$

The following technical conditions are needed to ensure that the decomposition $\mathbf{z}_{t}=$ $\chi_{t}+\mathbf{e}_{t}$ is unique. See Theorem B of Forni et al. (2015b).

\section{Condition 3.}

(a) For each $m, \mathbf{z}_{t}$ is a second order $m$-dimensional stationary process that has a spectral density.

(b) Let $\mathbf{\Sigma}(0)$ be the covariance matrix of $\mathbf{z}_{t}$. Let $\lambda_{m, j}^{\mathbf{z}}$ be its $j$-th eigenvalue and let $\lambda_{j}^{\mathbf{z}}=\sup _{m \in \mathbb{N}} \lambda_{m, j}^{\mathbf{z}}$. Then $\lambda_{k_{2}+1}^{\mathbf{z}}=\infty$ and $\lambda_{k_{2}+2}^{\mathbf{z}}<\infty$.

If we further assume that the idiosyncratic disturbances are cross-sectionally uncorrelated, we can prove that the ODPC is able to recover the common part of the dynamic factor model asymptotically. 


\section{Condition 4.}

(a) There exists $L>0$ such that $\mathbb{E} z_{t, j}^{2} \leq L$ for all $j$.

(b) $\boldsymbol{\Sigma}^{e}(0)$ is a diagonal matrix.

Theorem 3. Assume Conditions 1, 2, 3 and 4 hold. Then as $m \rightarrow \infty$

$$
\frac{1}{m} \mathbb{E}\left\|\mathbf{B}^{\prime} \mathbf{f}_{t}-\widehat{\mathbf{z}}_{t}\right\|^{2} \rightarrow 0
$$

where $\widehat{\mathbf{z}}_{t}=\mathbf{z}_{t}^{R}\left(\mathbf{a}^{*}, \mathbf{B}^{*}\right)$, for $\left(\mathbf{a}^{*}, \mathbf{B}^{*}\right) \in \mathcal{I}$.

\section{Simulation study}

In this Section, we compare the procedure proposed in this paper (ODPC) with those of Forni et al. (2005) (FHLR), Forni et al. (2015b) (FHLZ) and Stock and Watson (2002) (SW) for forecasting multivariate time series.

We took $(T, m) \in\{50,100,200\} \times\{50,100,200\}$. This Monte Carlo design includes difficult forecasting situations where the ratio $T / m$ is smaller than one. For each combination of $T$ and $m$, we generated 500 vector time series with $T+1$ periods of the following models.

DFM1 The generating model is $z_{t, j}=c\left(\sin (2 \pi j / m) f_{t}+\cos (2 \pi j / m) f_{t-1}+(j / m) f_{t-2}+f_{t-3}\right)+$ $u_{t, j}$ where the $u_{t, j}$ are i.i.d. standard normal random variables. The factor $f_{t}$ is generated according to a moving average process $f_{t}=v_{t}+\theta_{1} v_{t-1}+\theta_{2} v_{t-2}$, where the $v_{t}$ are i.i.d. standard normals. $\theta_{2}$ is generated at random uniformly on the interval $(-0.7,0.7)$. Then $\theta_{1}$ is generated at random uniformly on the interval $\left(0,1-\left|\theta_{2}\right|\right)$. $c$ is chosen so that the mean empirical variance of the common part is equal to one. This is a stationary dynamic factor model with four static factors and one dynamic factor. 
DFM1AR This model adds idiosyncratic AR(1) structure to DFM1. For each $j, u_{t, j}$ follows an unit variance $\mathrm{AR}(1)$, where at each replication the autoregression coefficient is generated at random, with uniform distribution in $(-0.9,0.9)$.

DFM2 The model is now $z_{t, j}=c\left(\sin (2 \pi j / m) f_{t}+\cos (2 \pi j / m) f_{t-1}+(j / m) f_{t-2}\right)+u_{t, j}$ where the $u_{t, j}$ are i.i.d. standard normal random variables. The factor $f_{t}$ follows an autoregressive model $f_{t}=1.4 f_{t-1}-0.45 f_{t-2}+v_{t}$, where the $v_{t}$ are i.i.d. standard normals. As in model DFM1, $c$ is chosen so that the mean empirical variance of the common part is equal to one. This is a stationary dynamic factor model with three static factors and one dynamic factor.

DFM2AR As in model DFM1 we add idiosyncratic AR(1) structure to DFM2. Again $u_{t, j}$ follows an unit variance $\mathrm{AR}(1)$ and at each replication the parameter is chosen from an uniform distribution in $(-0.9,0.9)$.

VARMA We first generate $\mathbf{x}_{t}=\Lambda \mathbf{x}_{t-1}+\mathbf{u}_{t}$, where the $\mathbf{u}_{t}$ are i.i.d standard multivariate normal variables and $\boldsymbol{\Lambda}$ is generated at random for each replication, as a diagonal matrix where the elements of the diagonal are independent and generated at random with uniform distribution in $(-0.9,0.9)$. We then take $\mathbf{z}_{t}=\mathbf{M} \mathbf{x}_{t}$, where $\mathbf{M}$ is a lower triangular matrix of dimensions $m \times m$ filled with ones. The $\mathbf{z}_{t}$ follow a stationary VARMA model. Finally, we standardize the data so that is has empirical mean variance equal to one.

For each estimator, and each combination of $T$ and $m$, using periods $1, \ldots, T$ we compute a forecast of each time series at period $T+1$ and the corresponding prediction mean squared error (PMSE). For the dynamic factor models, we only compute a forecast of the common part, that is, we did not include any forecasting of the idiosyncratic component. We report the average PMSE over the 500 replications. 
The MATLAB code to compute FHLR was obtained from http://morgana.unimore.it/forni_mario The Bartlett lag-window size was taken as $[\sqrt{T+1}]$. The MATLAB code to compute FHLZ was kindly provided by the authors. We used a triangular kernel, with window size equal to $\left[(T+1)^{2 / 3}\right]$. The maximum order of the singular VARs was taken to be 5 , and the order was chosen using the BIC criterion. The number of random permutations of the series was taken to be 30 . We used our own implementation of the SW procedure. For FHLR and FHLZ, the procedures were applied to the data standardized to zero mean and unit variance. At the end, the forecasts were transformed to the original units. The SW forecast was obtained by projecting $\mathbf{z}_{T+1}$ on the estimated factors, as in equation 16 of Forni et al. (2005). For ODPC, to forecast future values of the dynamic principal components we use the auto.arima and forecast.arima functions from the forecast $\mathrm{R}$ package (Hyndman and Khandakar, 2008) to automatically fit (possibly seasonal) ARIMA models to the dynamic components and obtain their forecasts.

For the factor models we use the known number of factors and lags. For the ODPC procedure, we take one component with $k_{1}=k_{2}$, equal to the number of lags in the factors in the generated model. Results for each combination of $(T, m)$, are shown in Tables 1 and 2 .

For the VARMA, model we use three different combinations of number of components and lags. We have used one, two and five dynamic factors for ODPC, FHLR and FHLZ and the equivalent, or larger, number of static factors for SW. Results are shown in Table 3. The second rows show, for ODPC (number of components, $k_{1}, k_{2}$ ), for FHLR (number of dynamic factors, number of static factors), for FHLZ (number of dynamic factors) and for SW (number of static factors).

Highlighted in black is the best result. An asterisk indicates that the difference with the runner up is significant at the $95 \%$ level, taking the difference of the squared forecasting 
errors. Table 1 shows that for models DFM1 and DFM1AR, with MA factors, the method ODPC always works better than the competitors, although the differences tend to be small. The largest difference with respect to the runner-up in both models is around $10 \%$. Table 2 shows that for AR factors, models DFM2 and DFM2AR, the errors of all the methods are smaller, as expected, and ODPC performs similar to SW and slightly better than the others. However, note that ODPC makes forecasts with only one dynamic component whereas the SW procedure requires three or four static factors. In Table 3 again ODPC is most often the winner, although the differences with the runner-up are not large.

In summary, the proposed procedure seems to work well, both for dynamic factor models and for VARMA models with large common dependency. 


\begin{tabular}{|c|c|c|c|c|c|c|c|c|c|}
\hline & & DFM1 & & & & DFM1A & & & \\
\hline$T$ & $m$ & ODPC & FHLR & FHLZ & $\mathrm{SW}$ & ODPC & FHLR & FHLZ & SW \\
\hline \multirow[t]{3}{*}{50} & 50 & $1.460^{*}$ & 1.510 & 1.742 & 1.542 & 1.429 & 1.460 & 1.617 & 1.493 \\
\hline & 100 & $1.411^{*}$ & 1.454 & 1.637 & 1.533 & 1.469 & 1.495 & 1.595 & 1.535 \\
\hline & 200 & 1.436 & 1.446 & 1.643 & 1.527 & $1.413^{*}$ & 1.464 & 1.575 & 1.509 \\
\hline \multirow[t]{3}{*}{100} & 50 & $1.327^{*}$ & 1.460 & 1.569 & 1.489 & $1.341^{*}$ & 1.453 & 1.543 & 1.479 \\
\hline & 100 & $1.294^{*}$ & 1.367 & 1.512 & 1.439 & $1.306^{*}$ & 1.411 & 1.509 & 1.452 \\
\hline & 200 & $1.262^{*}$ & 1.330 & 1.494 & 1.378 & $1.310^{*}$ & 1.377 & 1.481 & 1.418 \\
\hline \multirow[t]{3}{*}{200} & 50 & $1.275^{*}$ & 1.373 & 1.484 & 1.413 & $1.271^{*}$ & 1.397 & 1.457 & 1.396 \\
\hline & 100 & $1.216^{*}$ & 1.313 & 1.403 & 1.365 & $1.283^{*}$ & 1.384 & 1.489 & 1.412 \\
\hline & 200 & $1.232^{*}$ & 1.302 & 1.448 & 1.360 & $1.240^{*}$ & 1.316 & 1.413 & 1.370 \\
\hline
\end{tabular}

Table 1: Means of the 1-step ahead PMSEs of ODPC, FHLR, FHLZ and SW for models DFM1 and DFM1AR. 


\begin{tabular}{llllllllll}
\hline \multicolumn{3}{c}{ DFM2 } & \multicolumn{7}{c}{ DFM2AR } \\
\hline$T$ & $m$ & ODPC & FHLR & FHLZ & SW & ODPC & FHLR & FHLZ & SW \\
\hline 50 & 50 & 1.268 & 1.286 & 1.712 & $\mathbf{1 . 2 4 3} *$ & $\mathbf{1 . 2 0 5}$ & 1.269 & 1.757 & 1.208 \\
& 100 & $\mathbf{1 . 2 1 7}$ & 1.277 & 1.594 & 1.219 & 1.199 & 1.238 & 1.582 & $\mathbf{1 . 1 8 4}$ \\
& 200 & $\mathbf{1 . 1 8 5 *}$ & 1.271 & 1.525 & 1.210 & $\mathbf{1 . 1 6 5}$ & 1.243 & 1.520 & 1.174 \\
100 & 50 & 1.149 & 1.146 & 1.357 & $\mathbf{1 . 1 2 8 *}$ & 1.132 & 1.147 & 1.375 & $\mathbf{1 . 1 2 3}$ \\
& 100 & $\mathbf{1 . 1 1 9}$ & 1.151 & 1.322 & 1.124 & 1.110 & 1.136 & 1.309 & $\mathbf{1 . 1 1 0}$ \\
& 200 & $\mathbf{1 . 1 0 3 *}$ & 1.136 & 1.265 & 1.112 & $\mathbf{1 . 0 9 7}$ & 1.127 & 1.280 & 1.100 \\
200 & 50 & 1.110 & 1.095 & 1.202 & $\mathbf{1 . 0 8 6 *}$ & 1.120 & 1.113 & 1.260 & $\mathbf{1 . 0 9 7}$ \\
& 100 & 1.092 & 1.087 & 1.174 & $\mathbf{1 . 0 7 8 *}$ & 1.083 & 1.089 & 1.201 & $\mathbf{1 . 0 7 6 *}$ \\
& 200 & $\mathbf{1 . 0 7 3 *}$ & 1.089 & 1.174 & 1.079 & $\mathbf{1 . 0 6 3}$ & 1.082 & 1.188 & 1.066 \\
\hline
\end{tabular}

Table 2: Means of the 1-step ahead PMSEs of ODPC, FHLR, FHLZ and SW for models DFM2 and DFM2AR. 


\begin{tabular}{|c|c|c|c|c|c|c|c|c|c|c|c|c|c|}
\hline$T$ & $m$ & ODPC & FHLR & FHLZ & SW & ODPC & FHLR & FHLZ & SW & ODPC & FHLR & FHLZ & SW \\
\hline & & $(1,1,1)$ & $(1,2)$ & (1) & $(2)$ & $(2,1,1)$ & $(2,6)$ & (2) & (6) & $(5,1,1)$ & $(5,10)$ & $(5)$ & (10) \\
\hline \multirow[t]{3}{*}{50} & 50 & 0.997 & 1.024 & 1.042 & 1.042 & 1.010 & 1.017 & 0.978 & 1.052 & 1.020 & 0.995 & 0.972 & 1.029 \\
\hline & 100 & 1.001 & 0.992 & 1.025 & 1.007 & 1.010 & 1.027 & 0.972 & 1.049 & 1.041 & 1.026 & $0.972 *$ & 1.060 \\
\hline & 200 & 0.999 & 1.047 & 1.015 & 1.050 & 1.010 & 1.034 & 0.974 & 1.063 & 1.017 & 1.035 & 0.996 & 1.079 \\
\hline \multirow[t]{3}{*}{100} & 50 & 0.863 & 0.909 & 0.891 & 0.926 & 0.854 & 0.858 & 0.862 & 0.900 & 0.854 & 0.835 & 0.872 & 0.866 \\
\hline & 100 & $0.926^{*}$ & 1.000 & 0.976 & 1.021 & 0.918 & 0.986 & 0.933 & 1.013 & 0.920 & 0.956 & 0.959 & 0.991 \\
\hline & 200 & $0.938^{*}$ & 1.038 & 0.993 & 1.021 & 0.933 & 1.041 & 0.943 & 1.071 & $0.933^{*}$ & 1.031 & 1.006 & 1.044 \\
\hline \multirow[t]{3}{*}{200} & 50 & 0.856 & 0.898 & 0.905 & 0.906 & 0.844 & 0.840 & 0.833 & 0.880 & 0.836 & 0.811 & 0.860 & 0.851 \\
\hline & 100 & $0.826 *$ & 0.951 & 0.884 & 0.963 & 0.818 & 0.932 & 0.832 & 0.954 & $0.812^{*}$ & 0.912 & 0.887 & 0.921 \\
\hline & 200 & $0.893^{*}$ & 0.996 & 0.950 & 0.997 & 0.878 & 0.969 & 0.882 & 0.995 & $0.873^{*}$ & 0.973 & 0.919 & 0.976 \\
\hline
\end{tabular}

Table 3: Means of the 1-step ahead PMSEs of ODPC, FHLR, FHLZ and SW for the VARMA model. 


\section{An empirical example}

In this section, we compare the forecasting performances of the procedures considered in the previous section when applied to a panel of real macroeconomic variables. The data set, downloaded from https://research.stlouisfed.org/econ/mccracken/fred-databases/, consists of several key monthly macroeconomic variables for the US economy used by Stock and Watson (2002) . A full description of the data can be found in the website. See also McCracken and Ng (2016). The data was corrected for outliers and transformed to stationarity using the MATLAB script provided in the aforementioned website. We kept only periods from January 1960 to February 2014 and removed series with missing data, resulting in a balanced panel with $T=650$ observations on $m=94$ series. Let $\mathbf{Z}=\left\{z_{t, j}\right\}$ be the resulting panel.

Following McCracken and $\mathrm{Ng}(2016)$ we used four series as target variables for forecasting: CLAIMSx, initial jobless claims, S\&P: indust, the S\&P Industrial Index, M2REAL, Real M2 Money Stock and INDPRO, Industrial Production Index, all in log levels. Let $j$ be the index of any of the target variables. Since the targets are transformed by taking first differences of the logarithm, the target at time $t+h$ is $z_{t+1, j}+\cdots+z_{t+h, j}$.

We considered one and two years forecast horizons, $h=12,24$, and selected the most recent out of sample forecast period of McCracken and $\mathrm{Ng}$ (2016), the one going from 2008:01 to 2014:12. Thus, we fit the four procedures discussed in the previous Monte Carlo section using sample periods $1, \ldots,(T-h-t)$ for each $t=0, \ldots, 83$ to predict $T-t$ that is, we use a rolling seven year window, covering the recovery of the US economy. We compute the $h$-steps ahead forecasts of the whole panel, and then compare the predicted values of the target variable with the actual values. Let

$$
E_{t, h}=\left(z_{T-t-h+1,6}+\cdots+z_{T-t, 6}-\left(\widehat{z}_{T-t-h+1,6 \mid T-h-t}+\cdots+\widehat{z}_{T-t, 6 \mid T-h-t}\right)\right)
$$


be the forecasting error of period $T-t$ using information up to period $T-h-t$, for $t=0, \ldots, 83$. We measure the performance of each procedure by

$$
\left(\frac{1}{84} \sum_{t=0}^{83} E_{t, h}^{2}\right)^{1 / 2}
$$

As in McCracken and $\mathrm{Ng}$ (2016) we compare the forecasts obtained by the different procedures using the first factor with lags. Thus, we did not try to forecast the idiosyncratic part, as our objective is to compare the performance of the methods in forecasting the common component in the series. We computed: ODPC with one component and up to three lags, FHLR with one dynamic factor and up to four static factors (this amount to assuming that the dynamic factor is loaded with up to three lags), FHLZ with one dynamic factor and SW with one static factor and up to three of its lags. We also computed a one-dimensional SARIMA forecast, by automatically fitting a SARIMA model using the auto.arima function from the forecast $\mathrm{R}$ package, using the default settings.

We report the root means squared forecasting errors relative to those of the onedimensional SARIMA forecast. Results are shown in Table 4. For a one year horizon, $h=12$, in half of the four series the best forecast is obtained with ODPC, that reduces the univariate forecast errors by $12.1 \%$ in CLAIMSx, and by $2.9 \%$ in S\&P Indust. The largest reduction of error with respect to the univariate forecast is in M2REAL, where all the procedures reduce the forecast error between $14,9 \%$ and $11,9 \%$ and the winner in this case is SW. For INDPRO the maximum error reduction is $8,5 \%$ and is obtained by FHLR. For the two year horizon, $h=24$, for three of the four series, the best forecast is obtained with ODPC, reducing the univariate forecast errors by $28.8 \%$ in CLAIMSx, $10.3 \%$ in S\&P indust and $13.2 \%$ in INDPRO. For M2REAL the best forecast is again obtained with SW, achieving a $19.8 \%$ reduction in error with respect to the univariate method. The conclusion is that the results of the four precedures in this data set are similar with a small advantage 
of ODPC. 


\begin{tabular}{lllll}
\hline & CLAIMSx & S\&P Indust & M2REAL & INDPRO \\
\hline $\mathrm{h}=12$ & & & & \\
\hline ODPC 1 & $\mathbf{0 . 8 7 9}$ & $\mathbf{0 . 9 7 1}$ & 0.901 & 1.001 \\
ODPC 2 & 0.894 & 0.972 & 0.891 & 1.025 \\
ODPC 3 & 0.913 & 0.981 & 0.869 & 1.041 \\
FHLR 1 & 1.003 & 1.019 & 0.881 & 0.944 \\
FHLR 2 & 0.904 & 0.997 & 0.897 & 0.931 \\
FHLR 3 & 0.920 & 1.006 & 0.903 & $\mathbf{0 . 9 1 5}$ \\
FHLZ & 0.994 & 1.003 & 0.868 & 0.960 \\
SW 1 & 1.004 & 1.006 & 0.853 & 1.033 \\
SW 2 & 1.018 & 1.018 & 0.852 & 1.046 \\
SW 3 & 1.028 & 1.029 & $\mathbf{0 . 8 5 1}$ & 1.058 \\
\hline h = 24 & & & & \\
\hline ODPC 1 & $\mathbf{0 . 7 1 2}$ & 0.900 & 0.932 & $\mathbf{0 . 8 6 8}$ \\
ODPC 2 & 0.722 & $\mathbf{0 . 8 9 7}$ & 0.930 & 0.886 \\
ODPC 3 & 0.729 & 0.908 & 0.897 & 0.903 \\
FHLR 1 & 1.006 & 1.003 & 0.848 & 0.933 \\
FHLR 2 & 0.877 & 0.974 & 0.855 & 0.888 \\
FHLR 3 & 0.892 & 0.982 & 0.849 & 0.883 \\
FHLZ & 1.002 & 0.994 & 0.855 & 0.938 \\
SW 1 & 1.086 & 1.056 & 0.803 & 1.035 \\
SW 2 & 1.101 & 1.067 & $\mathbf{0 . 8 0 2}$ & 1.047 \\
SW 3 & 1.116 & 1.078 & 0.802 & 1.058 \\
\hline
\end{tabular}

Table 4: RMSE forecasting errors for different number of lags, relative to the RMSE of the one dimensional SARIMA forecast. 


\section{Choosing the number of components and lags}

In practice, the number of components and lags needs to be chosen. To simplify the notation, assume that for each component $k_{1}^{i}=k_{2}^{i}$, that is, the number of lags of $\mathbf{z}_{t}$ used to define the dynamic principal component and the number of lags of $\widehat{f}_{t}$ used to reconstruct the original series are the same.

One possible approach is to minimize the cross-validated forecasting error in a stepwise fashion. Choose a maximum number of lags $K_{\text {max }}$, and, starting with one component, search for the value $k^{*}$ among $0, \ldots, K_{\max }$ that gives the minimum cross-validated forecasting error. Then, fix the first component computed with $k^{*}$ lags and repeat the procedure with the second component. If the optimal cross-validated forecasting error using the two components is larger than the one using only one component, stop; otherwise add a third component and proceed as before.

The same stepwise approach could be applied to minimize an information criterion. This would reduce the computational burden significantly. The following BIC type criterion could be used. Suppose we have computed $q$ dynamic principal components, each with $k_{1}^{i}=$ $k_{2}^{i}=k^{i}$ lags. Let $\widehat{y}_{t, j}=\widehat{\alpha}_{j}+\sum_{h=0}^{k^{q}} \widehat{\beta}_{h, j} \widehat{f}_{t-h}, t=2 \sum_{i=1}^{q} k^{i}+1, \ldots, T$ be the reconstruction obtained, where $y_{t, j}=z_{t, j}$ for the first component and will be equal to the residuals from the fit with the previous components otherwise. Let $r_{t, j}=y_{t, j}-\widehat{y}_{t, j}$ be the residuals, $\mathbf{R}_{q}$ be the corresponding matrix of residuals and $\boldsymbol{\Sigma}_{q}=\left(\mathbf{R}_{q}^{\prime} \mathbf{R}_{q}\right) /\left(T-2 \sum_{i=1}^{q} k^{i}\right)$. Then for each $q$ choose the value $k^{*}$ among $0, \ldots, K_{\max }$ that minimizes

$$
\mathrm{BIC}_{k}=\left(T-2 \sum_{i=1}^{q} k^{i}\right) \log \left(\operatorname{trace}\left(\boldsymbol{\Sigma}_{q}\right)\right)+m(2 k+3) \log \left(T-2 \sum_{i=1}^{q} k^{i}\right) .
$$

The performance of these alternatives will be the subject of further research. 


\section{Conclusions and possible extensions}

We have presented a new procedure for the dimension reduction of multivariate time series. The main advantages with respect to other alternatives are that: in the spirit of principal component analysis, it is not based on assuming any particular model (parametric or not) for the data, but rather on finding linear combinations of the observations with optimal reconstruction properties; not being based on both lags and leads of the data, it is useful for forecasting large sets of time series.

Moreover, the proposed procedure can be generalized in several directions. First, since the MSE criterion used in the is paper is not robust, it can substituted for the minimization of a robust scale. This can be achieved in a similar way as in Peña and Yohai (2016). A simpler way to obtain robustness would be to substitute the alternating least squares regressions by robust regression estimators, for example MM-estimators (Yohai, 1987). Second, to deal with very large number of variables the estimation algorithm can be regularized. For example, in each of the steps, the alternating least squares regressions may be replaced by a regularized regressions, using, for example, a Lasso procedure. This method will allow for a different number of lags in different variables. Both modifications, for robustness and regularization, can be combined using in each step a robust lasso procedure (see for example Smucler and Yohai (2017)). All these extensions require further research.

\section{Appendix}

This section includes the proofs of all the results stated in the paper. 


\section{Lemma 1.}

$$
\sup _{\|\mathbf{a}\|=1}\left\|\frac{\mathbf{F}_{k_{1}, k_{2}}^{\prime} \mathbf{F}_{k_{1}, k_{2}}}{T-\left(k_{1}+k_{2}\right)}-\mathcal{S}(\mathbf{a})\right\|_{F} \stackrel{a . s .}{\rightarrow} 0 .
$$

Proof of Lemma 1. Fix a with $\|\mathbf{a}\|=1$. Then

$$
\begin{aligned}
& \frac{\mathbf{F}_{k_{1}, k_{2}}^{\prime} \mathbf{F}_{k_{1}, k_{2}}}{T-\left(k_{1}+k_{2}\right)} \\
& =\frac{1}{T-\left(k_{1}+k_{2}\right)}\left(\begin{array}{c}
\mathbf{1}_{T-\left(k_{1}+k_{2}\right)}^{\prime} \\
\mathbf{a}^{\prime} \mathbf{Z}_{k_{1}+k_{2}, 0}^{\prime} \\
\mathbf{a}^{\prime} \mathbf{Z}_{k_{1}+k_{2}-1,0}^{\prime} \\
\vdots \\
\mathbf{a}^{\prime} \mathbf{Z}_{k_{1}, 0}^{\prime}
\end{array}\right)\left(\begin{array}{lllll}
\mathbf{1}_{T-\left(k_{1}+k_{2}\right)} & \mathbf{Z}_{k_{1}+k_{2}, 0} \mathbf{a} & \mathbf{Z}_{k_{1}+k_{2}-1,0} \mathbf{a} & \ldots & \mathbf{Z}_{k_{1}, 0} \mathbf{a}
\end{array}\right)
\end{aligned}
$$

Fix $k_{1} \leq i, j \leq k_{1}+k_{2}$. Then

$$
\begin{aligned}
\mathbf{Z}_{i, 0}^{\prime} \mathbf{Z}_{j, 0} & =\left(\begin{array}{c}
\mathbf{Z}_{i}^{\prime} \\
\mathbf{Z}_{i-1}^{\prime} \\
\vdots \\
\mathbf{Z}_{i-k_{1}}^{\prime}
\end{array}\right)\left(\begin{array}{llll}
\mathbf{Z}_{j} & \mathbf{Z}_{j-1} & \ldots & \mathbf{Z}_{j-k_{1}}
\end{array}\right) \\
& =\left(\begin{array}{cccc}
\mathbf{Z}_{i}^{\prime} \mathbf{Z}_{j} & \mathbf{Z}_{i}^{\prime} \mathbf{Z}_{j-1} & \ldots & \mathbf{Z}_{i}^{\prime} \mathbf{Z}_{j-k_{1}} \\
\vdots & \vdots & & \vdots \\
\mathbf{Z}_{i-k_{1}}^{\prime} \mathbf{Z}_{j} & \mathbf{Z}_{i-k_{1}}^{\prime} \mathbf{Z}_{j-1} & \ldots & \mathbf{Z}_{i-k_{1}}^{\prime} \mathbf{Z}_{j-k_{1}}
\end{array}\right) .
\end{aligned}
$$

Note that

$$
\mathbf{Z}_{i}^{\prime} \mathbf{Z}_{j}=\sum_{r=1}^{T-\left(k_{1}+k_{2}\right)} \mathbf{z}_{i+r} \mathbf{z}_{j+r}^{\prime}
$$

By the Ergodic Theorem

$$
\frac{\mathbf{Z}_{i}^{\prime} \mathbf{Z}_{j}}{T-\left(k_{1}+k_{2}\right)} \stackrel{\text { a.s. }}{\rightarrow} \mathbf{\Sigma}(i-j) .
$$


Hence

$$
\frac{\mathbf{Z}_{i, 0}^{\prime} \mathbf{Z}_{j, 0}}{T-\left(k_{1}+k_{2}\right)} \stackrel{\text { a.s. }}{\rightarrow}\left(\begin{array}{cccc}
\Sigma(i-j) & \Sigma(i-j+1) & \ldots & \boldsymbol{\Sigma}\left(i-j+k_{1}\right) \\
\vdots & \vdots & & \vdots \\
\Sigma\left(i-j-k_{1}\right) & \boldsymbol{\Sigma}\left(i-j-k_{1}+1\right) & \ldots & \boldsymbol{\Sigma}(i-j)
\end{array}\right)=\mathbf{V}(i-j) .
$$

On the other hand

$$
\begin{aligned}
\frac{1}{T-\left(k_{1}+k_{2}\right)} \mathbf{a}^{\prime} \mathbf{Z}_{i, 0}^{\prime} \mathbf{1}_{T-\left(k_{1}+k_{2}\right)} & =\frac{1}{T-\left(k_{1}+k_{2}\right)} \sum_{h=0}^{k_{1}} \mathbf{a}_{h}^{\prime} \mathbf{Z}_{i-h}^{\prime} \mathbf{1}_{T-\left(k_{1}+k_{2}\right)} \\
& =\frac{1}{T-\left(k_{1}+k_{2}\right)} \sum_{h=0}^{k_{1}} \mathbf{a}_{h}^{\prime}\left(\begin{array}{c}
\sum_{r=1}^{T-\left(k_{1}+k_{2}\right)} z_{i-h+r, 1} \\
\vdots \\
T-\left(k_{1}+k_{2}\right) \\
\sum_{r=1} z_{i-h+r, m}
\end{array}\right) \stackrel{\text { a.s. }}{\rightarrow} 0,
\end{aligned}
$$

by the Ergodic Theorem and since $\mathbb{E} \mathbf{z}_{t}=0$ by assumption. We have shown that

$$
\frac{\mathbf{F}_{k_{1}, k_{2}}^{\prime} \mathbf{F}_{k_{1}, k_{2}}}{T-\left(k_{1}+k_{2}\right)} \stackrel{a . s .}{\rightarrow}\left(\begin{array}{ccccc}
1 & 0 & 0 & \ldots & 0 \\
0 & \mathbf{a}^{\prime} \mathbf{V}(0) \mathbf{a} & \mathbf{a}^{\prime} \mathbf{V}(1) \mathbf{a} & \ldots & \mathbf{a}^{\prime} \mathbf{V}\left(k_{2}\right) \mathbf{a} \\
\vdots & \vdots & \vdots & & \vdots \\
0 & \mathbf{a}^{\prime} \mathbf{V}\left(-k_{2}\right) \mathbf{a} & \mathbf{a}^{\prime} \mathbf{V}\left(-k_{2}+1\right) \mathbf{a} & \ldots & \mathbf{a}^{\prime} \mathbf{V}(0) \mathbf{a}
\end{array}\right)=\mathcal{S}(\mathbf{a}) .
$$

To prove that the convergence holds uniformly, it suffices to show that, for $k_{1} \leq i, j \leq$ $k_{1}+k_{2}$,

$$
\begin{aligned}
& \sup _{\|\mathbf{a}\|=1}\left\|\frac{1}{T-\left(k_{1}+k_{2}\right)} \sum_{h=0}^{k_{1}} \mathbf{a}_{h}^{\prime} \mathbf{Z}_{i-h}^{\prime} \mathbf{1}_{T-\left(k_{1}+k_{2}\right)}\right\| \stackrel{\text { a.s. }}{\rightarrow} 0 \text { and } \\
& \sup _{\|\mathbf{a}\|=1}\left|\frac{\mathbf{a}^{\prime} \mathbf{Z}_{i, 0}^{\prime} \mathbf{Z}_{j, 0} \mathbf{a}}{T-\left(k_{1}+k_{2}\right)}-\mathbf{a}^{\prime} \mathbf{V}(i-j) \mathbf{a}\right| \stackrel{\text { a.s. }}{\rightarrow} 0
\end{aligned}
$$

The first assertion follows immediately from the Ergodic Theorem. It is easy to show that

$$
\frac{\mathbf{a}^{\prime} \mathbf{Z}_{i, 0}^{\prime} \mathbf{Z}_{j, 0} \mathbf{a}}{T-\left(k_{1}+k_{2}\right)}=\frac{\sum_{h=0}^{k_{1}} \sum_{r=0}^{k_{1}} \mathbf{a}_{r}^{\prime} \mathbf{Z}_{i-r}^{\prime} \mathbf{Z}_{j-h} \mathbf{a}_{h}}{T-\left(k_{1}+k_{2}\right)} \text {. }
$$


Note that for any $\mathbf{v}, \mathbf{w} \in \mathbb{R}^{m}$

$$
\mathbf{v}^{\prime} \mathbf{Z}_{i}^{\prime} \mathbf{Z}_{j} \mathbf{w}=\mathbf{v}^{\prime}\left(\sum_{r=1}^{T-\left(k_{1}+k_{2}\right)} \mathbf{z}_{i+r} \mathbf{z}_{j+r}^{\prime}\right) \mathbf{w}
$$

Thus, to prove the lemma it will be enough to prove that

$$
\sup _{\|\mathbf{v}\| \leq 1,\|\mathbf{w}\| \leq 1}\left|\frac{\mathbf{v}^{\prime}\left(\sum_{r=1}^{T-\left(k_{1}+k_{2}\right)} \mathbf{z}_{i+r} \mathbf{z}_{j+r}^{\prime}\right) \mathbf{w}}{T-\left(k_{1}+k_{2}\right)}-\mathbf{v}^{\prime} \mathbf{\Sigma}(i-j) \mathbf{w}\right| \stackrel{\text { a.s. }}{\rightarrow} 0 .
$$

This follows immediately from

$$
\sup _{\|\mathbf{v}\| \leq 1\|\mathbf{w}\| \leq 1}\left|\frac{\mathbf{v}^{\prime}\left(\sum_{r=1}^{T-\left(k_{1}+k_{2}\right)} \mathbf{z}_{i+r} \mathbf{z}_{j+r}^{\prime}\right) \mathbf{w}}{T-\left(k_{1}+k_{2}\right)}-\mathbf{v}^{\prime} \boldsymbol{\Sigma}(i-j) \mathbf{w}\right| \leq\left\|\frac{\sum_{r=1}^{T-\left(k_{1}+k_{2}\right)} \mathbf{z}_{i+r} \mathbf{z}_{j+r}^{\prime}}{T-\left(k_{1}+k_{2}\right)}-\boldsymbol{\Sigma}(i-j)\right\|
$$

and the Ergodic Theorem.

\section{Lemma 2.}

$$
\liminf _{T} \inf _{\|\mathbf{a}\|=1} \lambda_{\min }\left(\frac{\mathbf{F}_{k_{1}, k_{2}}^{\prime} \mathbf{F}_{k_{1}, k_{2}}}{T-\left(k_{1}+k_{2}\right)}\right) \geq \inf _{\|\mathbf{a}\|=1} \lambda_{\min }(\mathcal{S}(\mathbf{a})),
$$

with probability one.

Proof of Lemma 2. It suffices to show that

$$
\sup _{\|\mathbf{a}\|=1}\left|\lambda_{\min }\left(\frac{\mathbf{F}_{k_{1}, k_{2}}^{\prime} \mathbf{F}_{k_{1}, k_{2}}}{T-\left(k_{1}+k_{2}\right)}\right)-\lambda_{\min }(\mathcal{S}(\mathbf{a}))\right| \stackrel{\text { a.s. }}{\rightarrow} 0
$$

and this follows from Theorem 3.3.16 of Horn and Johnson (1994) and Lemma 1.

To ease the notation, we will note

$$
g(M)=\left(M\left(\inf _{\|\mathbf{a}\|=1} \lambda_{\min }(\mathcal{S}(\mathbf{a}))\right)^{1 / 2}-\left(\mathbb{E}\|\mathbf{z}\|^{2}\right)^{1 / 2}\right)^{2} .
$$


If Condition 11 holds, clearly $g(M) \rightarrow+\infty$ when $M \rightarrow+\infty$. The following Lemma is a key result.

Lemma 3. Assume Condition 1 holds. Then if $M>\left(\mathbb{E}\|\mathbf{z}\|^{2} / \inf _{\|\mathbf{a}\|=1} \lambda_{\min }(\mathcal{S}(\mathbf{a}))\right)^{1 / 2}$, with probability 1

$$
\liminf \inf _{T \mid \mathbf{a}\|=1,\| \mathbf{D} \|_{F} \geq M} M S E(\mathbf{a}, \mathbf{D}) \geq g(M) .
$$

Proof of Lemma [3. Note that the triangle inequality implies that

$$
\operatorname{MSE}(\mathbf{a}, \mathbf{D})^{1 / 2} \geq \frac{\left\|\mathbf{F}_{k_{1}, k_{2}} \mathbf{D}\right\|_{F}-\left\|\mathbf{Z}_{2 k}\right\|_{F}}{\sqrt{T-\left(k_{1}+k_{2}\right)}}=\left\|\frac{\mathbf{F}_{k_{1}, k_{2}}}{\sqrt{T-\left(k_{1}+k_{2}\right)}} \mathbf{D}\right\|_{F}-\left\|\frac{\mathbf{Z}_{k_{1}+k_{2}}}{\sqrt{T-\left(k_{1}+k_{2}\right)}}\right\|_{F} .
$$

We will bound the right hand side of the last inequality. It follows from the Ergodic

Theorem that

$$
\left\|\frac{\mathbf{Z}_{k_{1}+k_{2}}}{\sqrt{T-\left(k_{1}+k_{2}\right)}}\right\|_{F}^{2}=\frac{1}{T-\left(k_{1}+k_{2}\right)} \sum_{t=\left(k_{1}+k_{2}\right)+1}^{T}\left\|\mathbf{z}_{t}\right\|^{2} \stackrel{a . s .}{\rightarrow} \mathbb{E}\|\mathbf{z}\|^{2} .
$$

On the other hand

$$
\inf _{\|\mathbf{a}\|=1,\|\mathbf{D}\|_{F} \geq M}\left\|\frac{\mathbf{F}_{k_{1}, k_{2}}}{\sqrt{T-\left(k_{1}+k_{2}\right)}} \mathbf{D}\right\|_{F} \geq M \inf _{\|\mathbf{a}\|=1} \inf _{\|\mathbf{D}\|_{F}=1}\left\|\frac{\mathbf{F}_{k_{1}, k_{2}}}{\sqrt{T-\left(k_{1}+k_{2}\right)}} \mathbf{D}\right\|_{F} .
$$

Note that

$$
\begin{aligned}
\inf _{\|\mathbf{D}\|_{F}=1}\left\|\frac{\mathbf{F}_{k_{1}, k_{2}}}{\sqrt{T-\left(k_{1}+k_{2}\right)}} \mathbf{D}\right\|_{F} & =\inf _{\|\mathbf{D}\|_{F}=1}\left\|\operatorname{vec}\left(\frac{\mathbf{F}_{k_{1}, k_{2}}}{\sqrt{T-\left(k_{1}+k_{2}\right)}} \mathbf{D}\right)\right\| \\
& =\inf _{\|\mathbf{D}\|_{F}=1}\left\|\left(\mathbf{I}_{m} \otimes \frac{\mathbf{F}_{k_{1}, k_{2}}}{\sqrt{T-\left(k_{1}+k_{2}\right)}}\right) \operatorname{vec}(\mathbf{D})\right\| \\
& =\inf _{\|\mathbf{d}\|=1}\left\|\left(\mathbf{I}_{m} \otimes \frac{\mathbf{F}_{k_{1}, k_{2}}}{\sqrt{T-\left(k_{1}+k_{2}\right)}}\right) \mathbf{d}\right\| \\
& =\lambda_{\min }^{1 / 2}\left(\left(\mathbf{I}_{m} \otimes \frac{\mathbf{F}_{k_{1}, k_{2}}^{\prime}}{\sqrt{T-\left(k_{1}+k_{2}\right)}}\right)\left(\mathbf{I}_{m} \otimes \frac{\mathbf{F}_{k_{1}, k_{2}}}{\sqrt{T-\left(k_{1}+k_{2}\right)}}\right)\right) \\
& =\lambda_{\min }^{1 / 2}\left(\mathbf{I}_{m} \otimes \frac{\mathbf{F}_{k_{1}, k_{2}}^{\prime} \mathbf{F}_{k_{1}, k_{2}}}{T-\left(k_{1}+k_{2}\right)}\right)=\lambda_{\min }^{1 / 2}\left(\frac{\mathbf{F}_{k_{1}, k_{2}}^{\prime} \mathbf{F}_{k_{1}, k_{2}}}{T-\left(k_{1}+k_{2}\right)}\right) .
\end{aligned}
$$


Hence, by Lemma 2

$$
\liminf _{T} \inf _{\|\mathbf{a}\|=1,\|\mathbf{D}\|_{F} \geq M}\left\|\frac{\mathbf{F}_{k_{1}, k_{2}}}{\sqrt{T-\left(k_{1}+k_{2}\right)}} \mathbf{D}\right\|_{F} \geq M\left(\inf _{\|\mathbf{a}\|=1} \lambda_{\min }(\mathcal{S}(\mathbf{a}))\right)^{1 / 2} .
$$

It follows that if $M>\left(\mathbb{E}\|\mathbf{z}\|^{2} / \inf _{\|\mathbf{a}\|=1} \lambda_{\min }(\mathcal{S}(\mathbf{a}))\right)^{1 / 2}$

$$
\liminf \inf _{T} \inf _{\|=1,\| \mathbf{D} \|_{F} \geq M} \operatorname{MSE}(\mathbf{a}, \mathbf{D}) \geq g(M)
$$

Let $\mathbb{P}_{T}$ be the empirical probability measure that places mass $1 /\left(T-\left(k_{1}+k_{2}\right)\right)$ at $\mathbf{y}_{1}=\left(\mathbf{z}_{1}, \ldots, \mathbf{z}_{\left(k_{1}+k_{2}\right)+1}\right), \ldots, \mathbf{y}_{T-\left(k_{1}+k_{2}\right)}=\left(\mathbf{z}_{T-\left(k_{1}+k_{2}\right)}, \ldots, \mathbf{z}_{T}\right)$. The process $\left(\mathbf{y}_{t}\right)_{t}$ is strictly stationary and ergodic. Let $L_{\mathbf{a}, \mathbf{D}}\left(\mathbf{y}_{t}\right)=\left\|\mathbf{z}_{t+\left(k_{1}+k_{2}\right)}-\widehat{\mathbf{z}}_{t+\left(k_{1}+k_{2}\right)}\right\|^{2}$. It follows that

$$
\operatorname{MSE}(\mathbf{a}, \mathbf{D})=\frac{1}{T-\left(k_{1}+k_{2}\right)} \sum_{t=\left(k_{1}+k_{2}\right)+1}^{T}\left\|\mathbf{z}_{t}-\widehat{\mathbf{z}}_{t}\right\|^{2}=\mathbb{P}_{T} L_{\mathbf{a}, \mathbf{D}}
$$

Lemma 4. For each $M>0$

$$
\sup _{\|\mathbf{a}\|=1,\|\mathbf{D}\|_{F} \leq M}\left|\mathbb{P}_{T} L_{\mathbf{a}, \mathbf{D}}-\mathbb{P} L_{\mathbf{a}, \mathbf{D}}\right| \stackrel{\text { a.s. }}{\rightarrow} 0
$$

Proof of Lemma 4. Let

$$
\mathcal{L}=\left\{L_{\mathbf{a}, \mathbf{D}}(\cdot): \mathbf{a} \in \mathbb{R}^{m\left(k_{1}+1\right)},\|\mathbf{a}\|=1, \mathbf{D} \in \mathbb{R}^{\left(k_{2}+2\right) \times m},\|\mathbf{D}\|_{F} \leq M\right\}
$$

$\mathcal{L}$ is VC-major, since it is formed by polynomials of bounded degree. It has an integrable envelope, since $\mathbb{E}\|\mathbf{z}\|^{2}<+\infty$. Moreover, if we take $\mathcal{L}^{0}$ to be the subset of $\mathcal{L}$ formed by taking only $\mathbf{a} \in \mathbb{Q}^{m\left(k_{1}+1\right)}$ and $\mathbf{D} \in \mathbb{Q}^{m \times\left(k_{2}+2\right)}$ it follows that: $\mathcal{L}^{0}$ is countable, and each element of $\mathcal{L}$ is the pointwise limit of elements of $\mathcal{L}^{0}$. Then the lemma follows from Proposition 1 of Adams and Nobel (2010). 
Lemma 5. Assume Condition 1 holds. Let $(\widetilde{\mathbf{a}}, \widetilde{\mathbf{D}})$ be such that $\|\widetilde{\mathbf{a}}\|=1$ and $M S E(\widetilde{\mathbf{a}}, \widetilde{\mathbf{D}}) \leq$ $\operatorname{MSE}(\widetilde{\mathbf{a}}, \mathbf{0})$ for all T. Fix $M_{0}$ such that

$$
M_{0} / 2>\left(\mathbb{E}\|\mathbf{z}\|^{2} / \inf _{\|\mathbf{a}\|=1} \lambda_{\min }(\mathcal{S}(\mathbf{a}))\right)^{1 / 2}
$$

and $g\left(M_{0} / 2\right)>\sup _{\|\mathbf{a}\|=1} M S E_{0}(\mathbf{a}, \mathbf{0})$. Then

$$
\mathbb{P}\left(\underset{T}{\limsup }\|\widetilde{\mathbf{D}}\|_{F}<M_{0}\right)=1 .
$$

Proof of Lemma囵. Let

$$
\begin{array}{r}
C=\left\{\limsup _{T} \sup _{\|\mathbf{a}\|=1,\|\mathbf{D}\|_{F}<M_{0}}\left|\mathbb{P}_{T} L_{\mathbf{a}, \mathbf{D}}-\mathbb{P} L_{\mathbf{a}, \mathbf{D}}\right|=0\right\}, \\
D=\left\{\liminf _{T} \inf _{\|\mathbf{a}\|=1,\|\mathbf{D}\|_{F} \geq M_{0} / 2} \operatorname{MSE}(\mathbf{a}, \mathbf{D}) \geq g\left(M_{0} / 2\right)\right\}, \\
E=\left\{\limsup _{T}\|\widetilde{\mathbf{D}}\|_{F} \geq M_{0}\right\} .
\end{array}
$$

Assume $\mathbb{P}(E)>0$. Then, by Lemmas 3 and $4, \mathbb{P}(C \cap D \cap E)>0$. Assume in what follows that we are working in the set $C \cap D \cap E$. Then for sufficiently large $T$

$$
\mathbb{P}_{T} L_{\widetilde{\mathbf{a}}, \mathbf{0}} \geq \mathbb{P}_{T} L_{\widetilde{\mathbf{a}}, \widetilde{\mathbf{D}}} \geq \inf _{\|\mathbf{a}\|=1,\|\mathbf{D}\|_{F} \geq M_{0} / 2} \mathbb{P}_{T} L_{\mathbf{a}, \mathbf{D}}
$$

It follows that

$$
\underset{T}{\limsup } \mathbb{P}_{T} L_{\widetilde{\mathbf{a}} \mathbf{0}} \geq \liminf _{T} \inf _{\|\mathbf{a}\|=1,\|\mathbf{D}\|_{F} \geq M_{0} / 2} \mathbb{P}_{T} L_{\mathbf{a}, \mathbf{D}} \geq g\left(M_{0} / 2\right) .
$$

It follows easily from $\limsup _{T} \sup _{\|\mathbf{a}\|=1,\|\mathbf{D}\|_{F}<M_{0}}\left|\mathbb{P}_{T} L_{\mathbf{a}, \mathbf{D}}-\mathbb{P} L_{\mathbf{a}, \mathbf{D}}\right|=0$ that

$$
\sup _{\|\mathbf{a}\|=1} \operatorname{MSE}_{0}(\mathbf{a}, \mathbf{0})=\sup _{\|\mathbf{a}\|=1} \mathbb{P} L_{\mathbf{a}, \mathbf{0}} \geq \limsup _{T} \mathbb{P}_{T} L_{\widetilde{\mathbf{a}}, \mathbf{0}} .
$$

But by assumption

$$
\sup _{\|\mathbf{a}\|=1} \operatorname{MSE}_{0}(\mathbf{a}, \mathbf{0})<g\left(M_{0} / 2\right) .
$$

We have arrived at a contradiction. It must be that $\mathbb{P}(E)=0$. 
Proof of Proposition 1. Take $M>0$ such that

$$
g(M)>2 \inf _{\|\mathbf{a}\|=1} \operatorname{MSE}(\mathbf{a}, \mathbf{0})
$$

and $M>\left(\mathbb{E}\|\mathbf{z}\|^{2} / \inf _{\|\mathbf{a}\|=1} \lambda_{\min }(\mathcal{S}(\mathbf{a}))\right)^{1 / 2}$. Since $\operatorname{MSE}(\mathbf{a}, \mathbf{D})$ is continuous, it attains its minimum over the compact set $\left\{(\mathbf{a}, \mathbf{D}):\|\mathbf{a}\|=1,\|\mathbf{D}\|_{F} \leq M\right\}$. Moreover

$$
\min _{\|\mathbf{a}\|=1,\|\mathbf{D}\| \leq M} \operatorname{MSE}(\mathbf{a}, \mathbf{D}) \leq \inf _{\|\mathbf{a}\|=1} \operatorname{MSE}(\mathbf{a}, \mathbf{0})
$$

Let

$$
A=\left\{\liminf \inf _{\|\mathbf{a}\|=1,\|\mathbf{D}\|_{F} \geq M} \operatorname{MSE}(\mathbf{a}, \mathbf{D})>g(M)\right\} .
$$

By Lemma 3, $\mathbb{P}(A)=1$. Assume we are working in the event $A$ in what follows. Then, there exists $T_{0}$ such that for $T>T_{0}, \operatorname{MSE}(\mathbf{a}, \mathbf{D})>\inf _{\|\mathbf{a}\|=1} \operatorname{MSE}(\mathbf{a}, \mathbf{0})$ for all $\mathbf{a}, \mathbf{D}$ with $\|\mathbf{a}\|=1,\|\mathbf{D}\|_{F} \geq M$. Hence, for $T>T_{0}, \min _{\|\mathbf{a}\|=1,\|\mathbf{D}\| \leq M} \operatorname{MSE}(\mathbf{a}, \mathbf{D}) \leq \operatorname{MSE}(\widetilde{\mathbf{a}}, \widetilde{\mathbf{D}})$ for all $(\widetilde{\mathbf{a}}, \widetilde{\mathbf{D}})$ with $\|\widetilde{\mathbf{a}}\|=1$, from which the results follows.

Proof of Proposition 2. Fix a with $\|\mathbf{a}\|=1$ and D. Note that

$$
\operatorname{MSE}_{0}(\mathbf{a}, \mathbf{D})=\mathbb{E}\left\|\mathbf{z}_{t}-\widehat{\mathbf{z}}_{t}\right\|^{2}=\mathbb{E}\left\|\widehat{\mathbf{z}}_{t}\right\|^{2}+\mathbb{E}\left\|\mathbf{z}_{t}\right\|^{2}-2 \mathbb{E} \mathbf{z}_{t}^{\prime} \widehat{\mathbf{z}}_{t}
$$

Let $\mathbf{f}_{t}^{\prime}=\mathbf{f}_{t}^{\prime}(\mathbf{a})=\left(1, \mathbf{a}^{\prime} \mathbf{x}_{t}, \mathbf{a}^{\prime} \mathbf{x}_{t-1}, \ldots, \mathbf{a}^{\prime} \mathbf{x}_{t-k_{2}}\right)$. Note that $\widehat{\mathbf{z}}_{t}=\mathbf{D}^{\prime} \mathbf{f}_{t}$. Hence

$$
\mathbb{E}\left\|\widehat{\mathbf{z}}_{t}\right\|^{2}=\mathbb{E} \mathbf{f}_{t}^{\prime} \mathbf{D D}^{\prime} \mathbf{f}_{t}=\operatorname{Tr}\left(\mathbf{D} \mathbf{D}^{\prime} \mathcal{S}(\mathbf{a})\right)
$$

Since $\mathbf{D D}^{\prime}$ and $\mathcal{S}(\mathbf{a})-\mathbf{I}_{k_{2}+2} \inf _{\|\mathbf{a}\|=1} \lambda_{\min }(\mathcal{S}(\mathbf{a}))$ are symmetric and semi-positive definite we have that

$$
\operatorname{Tr}\left(\mathbf{D D}^{\prime} \mathcal{S}(\mathbf{a})\right) \geq \operatorname{Tr}\left(\mathbf{D D}^{\prime} \inf _{\|\mathbf{a}\|=1} \lambda_{\min }(\mathcal{S}(\mathbf{a}))\right)=\|\mathbf{D}\|_{F}^{2} \inf _{\|\mathbf{a}\|=1} \lambda_{\min }(\mathcal{S}(\mathbf{a})) .
$$

On the other hand, by the Cauchy-Schwartz inequality

$$
\mathbb{E}\left|\mathbf{z}_{t}^{\prime} \widehat{\mathbf{z}}_{t}\right| \leq\left(\mathbb{E}\left\|\mathbf{z}_{t}\right\|^{2}\right)^{1 / 2}\left(\mathbb{E}\left\|\widehat{\mathbf{z}}_{t}\right\|^{2}\right)^{1 / 2}
$$


Since $\mathbf{D D}^{\prime}$ and $\mathbf{I}_{k_{2}+2} \sup _{\|\mathbf{a}\|=1} \lambda_{\max }(\mathcal{S}(\mathbf{a}))-\mathcal{S}(\mathbf{a})$ are symmetric and semi-positive definite we have that

$$
\operatorname{Tr}\left(\mathbf{D D}^{\prime} \mathcal{S}(\mathbf{a})\right) \leq \operatorname{Tr}\left(\mathbf{D D}^{\prime} \sup _{\|\mathbf{a}\|=1} \lambda_{\max }(\mathcal{S}(\mathbf{a}))\right)=\|\mathbf{D}\|_{F}^{2} \sup _{\|\mathbf{a}\|=1} \lambda_{\max }(\mathcal{S}(\mathbf{a}))
$$

Hence,

$$
\left(\mathbb{E}\left\|\widehat{\mathbf{z}}_{t}\right\|^{2}\right)^{1 / 2} \leq\|\mathbf{D}\|_{F}\left(\sup _{\|\mathbf{a}\|=1} \lambda_{\max }(\mathcal{S}(\mathbf{a}))\right)^{1 / 2}
$$

It follows that

$$
\inf _{\|\mathbf{a}\|=1} \mathbb{E}\left\|\mathbf{z}_{t}-\widehat{\mathbf{z}}_{t}\right\|^{2} \geq\|\mathbf{D}\|_{F}^{2} \inf _{\|\mathbf{a}\|=1} \lambda_{\min }(\mathcal{S}(\mathbf{a}))+\mathbb{E}\left\|\mathbf{z}_{t}\right\|^{2}-2\left(\mathbb{E}\left\|\mathbf{z}_{t}\right\|^{2}\right)^{1 / 2}\|\mathbf{D}\|_{F}\left(\sup _{\|\mathbf{a}\|=1} \lambda_{\max }(\mathcal{S}(\mathbf{a}))\right)^{1 / 2}
$$

from which the proposition follows immediately.

Proof of Theorem 1. Fix $\varepsilon>0$. Let $A=\left\{\limsup _{T} d((\widehat{\mathbf{a}}, \widehat{\mathbf{D}}), \mathcal{I}) \geq \varepsilon\right\}$. We will show that $\mathbb{P}(A)=0$. Assume $\mathbb{P}(A)>0$. Take $\left(\mathbf{a}^{*}, \mathbf{D}^{*}\right) \in \mathcal{I}$. Fix $M_{0}$ large enough such that $M_{0} / 2$ satisfies the hypothesis of Lemma 5. Note that

$$
\inf \left\{\operatorname{MSE}_{0}(\mathbf{a}, \mathbf{D}):\|\mathbf{a}\|=1,\|\mathbf{D}\|_{F} \leq M_{0}, d((\mathbf{a}, \mathbf{D}), \mathcal{I}) \geq \varepsilon / 2\right\}>\operatorname{MSE}_{0}\left(\mathbf{a}^{*}, \mathbf{D}^{*}\right) .
$$

Since $\mathbb{P}_{T} L_{\widehat{\mathbf{a}}, \widehat{\mathbf{D}}} \leq \mathbb{P}_{T} L_{\mathbf{a}^{*}, \mathbf{D}^{*}}$, and by the Ergodic Theorem

$$
\mathbb{P}_{T} L_{\mathbf{a}^{*}, \mathbf{D}^{*}} \stackrel{\text { a.s. }}{\rightarrow} \operatorname{MSE}_{0}\left(\mathbf{a}^{*}, \mathbf{D}^{*}\right),
$$

we have that, with probability one

$$
\underset{T}{\limsup } \mathbb{P}_{T} L_{\widehat{\mathbf{a}}, \widehat{\mathbf{D}}} \leq \operatorname{MSE}_{0}\left(\mathbf{a}^{*}, \mathbf{D}^{*}\right) .
$$


Let

$$
\begin{aligned}
B & =\left\{\limsup _{T} \sup _{\|\mathbf{a}\|=1,\|\mathbf{D}\|_{F} \leq M_{0}}\left|\mathbb{P}_{T} L_{\mathbf{a}, \mathbf{D}}-\mathbb{P} L_{\mathbf{a}, \mathbf{D}}\right|=0\right\}, \\
C & =\left\{\underset{T}{\left.\limsup \|\widehat{\mathbf{D}}\|_{F}<M_{0} / 2\right\} .}\right. \\
D & =\left\{\underset{T}{\limsup } \mathbb{P}_{T} L_{\widehat{\mathbf{a}}, \widehat{\mathbf{D}}} \leq \mathrm{MSE}_{0}\left(\mathbf{a}^{*}, \mathbf{D}^{*}\right)\right\} .
\end{aligned}
$$

Then, by Lemmas 5 and $4, \mathbb{P}(A \cap B \cap C \cap D)>0$. Assume in what follows that we are working in the set $A \cap B \cap C \cap D$. Note that since

$$
\left\{(\mathbf{a}, \mathbf{D}):\|\mathbf{a}\|=1,\|\mathbf{D}\|_{F} \leq M_{0}, d((\mathbf{a}, \mathbf{D}), \mathcal{I}) \geq \varepsilon / 2\right\} \subseteq\left\{(\mathbf{a}, \mathbf{D}):\|\mathbf{a}\|=1,\|\mathbf{D}\|_{F} \leq M_{0}\right\}
$$

we have that

$$
\limsup _{T} \sup \left\{\left|\mathbb{P}_{T} L_{\mathbf{a}, \mathbf{D}}-\mathbb{P} L_{\mathbf{a}, \mathbf{D}}\right|: \mid\|\mathbf{a}\|=1,\|\mathbf{D}\|_{F} \leq M_{0}, d((\mathbf{a}, \mathbf{D}), \mathcal{I}) \geq \varepsilon / 2\right\}=0,
$$

and hence that

$$
\begin{aligned}
& \liminf _{T} \inf \left\{\mathbb{P}_{T} L_{\mathbf{a}, \mathbf{D}}:\|\mathbf{a}\|=1,\|\mathbf{D}\|_{F} \leq M_{0}, d((\mathbf{a}, \mathbf{D}), \mathcal{I}) \geq \varepsilon / 2\right\} \\
& \geq \inf \left\{\mathbb{P} L_{\mathbf{a}, \mathbf{D}}:\|\mathbf{a}\|=1,\|\mathbf{D}\|_{F} \leq M_{0}, d((\mathbf{a}, \mathbf{D}), \mathcal{I}) \geq \varepsilon / 2\right\}
\end{aligned}
$$

Since $\limsup _{T} d((\widehat{\mathbf{a}}, \widehat{\mathbf{D}}), \mathcal{I}) \geq \varepsilon$ and $\limsup _{T}\|\widehat{\mathbf{D}}\|_{F}<M_{0} / 2$, there exists a subsequence of $(\widehat{\mathbf{a}}, \widehat{\mathbf{D}})$, which in an abuse of notation we continue to call $(\widehat{\mathbf{a}}, \widehat{\mathbf{D}})$, such that $d((\widehat{\mathbf{a}}, \widehat{\mathbf{D}}), \mathcal{I})>\varepsilon / 2$ and $\|\widehat{\mathbf{D}}\|_{F} \leq M_{0}$ for all $T$. Then

$$
\begin{aligned}
\operatorname{MSE}_{0}\left(\mathbf{a}^{*}, \mathbf{D}^{*}\right) & \geq \liminf _{T} \mathbb{P}_{T} L_{\widehat{\mathbf{a}}, \widehat{\mathbf{B}}} \\
& \geq \liminf _{T} \inf \left\{\mathbb{P}_{T} L_{\mathbf{a}, \mathbf{D}}:\|\mathbf{a}\|=1,\|\mathbf{D}\|_{F} \leq M_{0}, d((\mathbf{a}, \mathbf{D}), \mathcal{I}) \geq \varepsilon / 2\right\} \\
& \geq \inf \left\{\mathbb{P} L_{\mathbf{a}, \mathbf{D}}:\|\mathbf{a}\|=1,\|\mathbf{D}\|_{F} \leq M_{0}, d((\mathbf{a}, \mathbf{D}), \mathcal{I}) \geq \varepsilon / 2\right\} \\
& =\inf \left\{\operatorname{MSE}_{0}(\mathbf{a}, \mathbf{D}):\|\mathbf{a}\|=1,\|\mathbf{D}\|_{F} \leq M_{0}, d((\mathbf{a}, \mathbf{D}), \mathcal{I}) \geq \varepsilon / 2\right\} \\
& >\operatorname{MSE}_{0}\left(\mathbf{a}^{*}, \mathbf{D}^{*}\right),
\end{aligned}
$$


a contradiction. It must be that $\mathbb{P}(A)=0$.

Proof of Theorem [2. For all $\mathbf{a} \in \mathbb{R}^{m\left(k_{1}+1\right)}$

$$
\begin{aligned}
& \widehat{\mathbf{f}}_{t}(\mathbf{a})=\left(\begin{array}{ccc}
\mathbf{z}_{t}^{\prime} & \cdots & \mathbf{z}_{t-k_{1}}^{\prime} \\
\vdots & \vdots & \vdots \\
\mathbf{z}_{t-k_{2}}^{\prime} & \cdots & \mathbf{z}_{t-k_{1}-k_{2}}^{\prime}
\end{array}\right) \mathbf{a} \\
& =\left(\begin{array}{ccc}
\mathbf{f}_{t}^{\prime} \mathbf{B} & \ldots & \mathbf{f}_{t-k_{1}}^{\prime} \mathbf{B} \\
\vdots & \vdots & \vdots \\
\mathbf{f}_{t-k_{2}}^{\prime} \mathbf{B} & \ldots & \mathbf{f}_{t-k_{1}-k_{2}}^{\prime} \mathbf{B}
\end{array}\right) \mathbf{a}+\left(\begin{array}{ccc}
\mathbf{e}_{t}^{\prime} & \ldots & \mathbf{e}_{t-k_{1}}^{\prime} \\
\vdots & \vdots & \vdots \\
\mathbf{e}_{t-k_{2}}^{\prime} & \ldots & \mathbf{e}_{t-k_{1}-k_{2}}^{\prime}
\end{array}\right) \mathbf{a} \\
& =\left(\begin{array}{ccc}
\mathbf{f}_{t}^{\prime} & \ldots & \mathbf{f}_{t-k_{1}}^{\prime} \\
\vdots & \vdots & \vdots \\
\mathbf{f}_{t-k_{2}}^{\prime} & \ldots & \mathbf{f}_{t-k_{1}-k_{2}}^{\prime}
\end{array}\right)\left(\mathbf{I}_{k_{1}+1} \otimes \mathbf{B}\right) \mathbf{a}+\left(\begin{array}{ccc}
\mathbf{e}_{t}^{\prime} & \ldots & \mathbf{e}_{t-k_{1}}^{\prime} \\
\vdots & \vdots & \vdots \\
\mathbf{e}_{t-k_{2}}^{\prime} & \ldots & \mathbf{e}_{t-k_{1}-k_{2}}^{\prime}
\end{array}\right) \mathbf{a} \\
& =\mathbf{F}_{t}\left(\mathbf{I}_{k_{1}+1} \otimes \mathbf{B}\right) \mathbf{a}+\mathbf{E}_{t} \mathbf{a} .
\end{aligned}
$$

Let $\widetilde{\mathbf{a}} \in \mathbb{R}^{m\left(k_{1}+1\right)}$ be defined by $\widetilde{\mathbf{a}}=\left(\left(1 /\left\|\mathbf{b}_{0}\right\|\right) \mathbf{b}_{0}^{\prime}, \mathbf{0}_{m}, \ldots, \mathbf{0}_{m}\right)^{\prime}$. Since $\left(\mathbf{a}^{*}, \mathbf{B}^{*}\right) \in \mathcal{I}$

$$
\begin{aligned}
\operatorname{MSE}_{0}\left(\mathbf{a}^{*}, \mathbf{B}^{*}\right) & \leq \operatorname{MSE}_{0}\left(\widetilde{\mathbf{a}}, \mathbf{B} /\left\|\mathbf{b}_{0}\right\|\right) \\
& =\mathbb{E}\left\|\mathbf{B}^{\prime} \mathbf{f}_{t}+\mathbf{e}_{t}-\left(\mathbf{B}^{\prime} /\left\|\mathbf{b}_{0}\right\|\right) \mathbf{F}_{t}\left(\mathbf{I}_{k_{1}+1} \otimes \mathbf{B}\right) \widetilde{\mathbf{a}}-\left(\mathbf{B}^{\prime} /\left\|\mathbf{b}_{0}\right\|\right) \mathbf{E}_{t} \widetilde{\mathbf{a}}\right\|^{2} \\
& =\mathbb{E}\left\|\mathbf{B}^{\prime} \mathbf{f}_{t}-\mathbf{B}^{\prime} \mathbf{F}_{t}\left(\mathbf{I}_{k_{1}+1} \otimes\left(\mathbf{B} /\left\|\mathbf{b}_{0}\right\|\right)\right) \widetilde{\mathbf{a}}\right\|^{2}+\mathbb{E}\left\|\mathbf{e}_{t}-\left(\mathbf{B}^{\prime} /\left\|\mathbf{b}_{0}\right\|\right) \mathbf{E}_{t} \widetilde{\mathbf{a}}\right\|^{2} \\
& +2 \mathbb{E}\left(\mathbf{B}^{\prime} \mathbf{f}_{t}-\mathbf{B}^{\prime} \mathbf{F}_{t}\left(\mathbf{I}_{k_{1}+1} \otimes\left(\mathbf{B} /\left\|\mathbf{b}_{0}\right\|\right)\right) \widetilde{\mathbf{a}}\right)^{\prime}\left(\mathbf{e}_{t}-\left(\mathbf{B}^{\prime} /\left\|\mathbf{b}_{0}\right\|\right) \mathbf{E}_{t} \widetilde{\mathbf{a}}\right) .
\end{aligned}
$$

By Condition 2b) and c), $\mathbb{E}\left(\mathbf{B}^{\prime} \mathbf{f}_{t}-\mathbf{B}^{\prime} \mathbf{F}_{t}\left(\mathbf{I}_{k_{1}+1} \otimes\left(\mathbf{B} /\left\|\mathbf{b}_{0}\right\|\right)\right) \widetilde{\mathbf{a}}\right)^{\prime}\left(\mathbf{e}_{t}-\left(\mathbf{B}^{\prime} /\left\|\mathbf{b}_{0}\right\|\right) \mathbf{E}_{t} \widetilde{\mathbf{a}}\right)=0$. 
By Condition $2 \mathrm{a}$ )

$$
\begin{aligned}
\mathbb{E}\left\|\mathbf{B}^{\prime} \mathbf{f}_{t}-\mathbf{B}^{\prime} \mathbf{F}_{t}\left(\mathbf{I}_{k_{1}+1} \otimes\left(\mathbf{B} /\left\|\mathbf{b}_{0}\right\|\right)\right) \widetilde{\mathbf{a}}\right\|^{2} & \leq\|\mathbf{B}\|^{2} \mathbb{E}\left\|\mathbf{f}_{t}-\mathbf{F}_{t}\left(\mathbf{I}_{k_{1}+1} \otimes\left(\mathbf{B} /\left\|\mathbf{b}_{0}\right\|\right)\right) \widetilde{\mathbf{a}}\right\|^{2} \\
& =O(m) \mathbb{E}\left\|\mathbf{f}_{t}-\mathbf{F}_{t}\left(\mathbf{I}_{k_{1}+1} \otimes\left(\mathbf{B} /\left\|\mathbf{b}_{0}\right\|\right)\right) \widetilde{\mathbf{a}}\right\|^{2}
\end{aligned}
$$

By Condition 2a), $\left\|\mathbf{b}_{0}\right\|^{2} / m \rightarrow 1$. Then

$$
\begin{aligned}
\left(\mathbf{I}_{k_{1}+1} \otimes\left(\mathbf{B} /\left\|\mathbf{b}_{0}\right\|\right)\right) \widetilde{\mathbf{a}} & =\left(1,\left(\mathbf{b}_{1}^{\prime} \mathbf{b}_{0}\right) /\left\|\mathbf{b}_{0}\right\|^{2},\left(\mathbf{b}_{2}^{\prime} \mathbf{b}_{0}\right) /\left\|\mathbf{b}_{0}\right\|^{2}, \ldots, 0\right) \\
& \rightarrow(1,0, \ldots, 0)^{\prime} \in \mathbb{R}^{\left(k_{1}+1\right)\left(k_{2}+1\right)}
\end{aligned}
$$

which implies that $\mathbf{F}_{t}\left(\mathbf{I}_{k_{1}+1} \otimes\left(\mathbf{B} /\left\|\mathbf{b}_{0}\right\|\right)\right) \widetilde{\mathbf{a}} \rightarrow \mathbf{f}_{t}$.

It follows from Condition $2 \mathrm{~b}$ ) and the Bounded Convergence Theorem that

$$
\frac{1}{m} \mathbb{E}\left\|\mathbf{B}^{\prime} \mathbf{f}_{t}-\mathbf{B}^{\prime} \mathbf{F}_{t}\left(\mathbf{I}_{k_{1}+1} \otimes\left(\mathbf{B} /\left\|\mathbf{b}_{0}\right\|\right)\right) \widetilde{\mathbf{a}}\right\|^{2} \rightarrow 0 .
$$

Note that

$$
\mathbb{E}\left\|\mathbf{e}_{t}-\left(\mathbf{B}^{\prime} /\left\|\mathbf{b}_{0}\right\|\right) \mathbf{E}_{t} \widetilde{\mathbf{a}}\right\|^{2}=\mathbb{E}\left\|\mathbf{e}_{t}\right\|^{2}+\mathbb{E}\left\|\left(\mathbf{B}^{\prime} /\left\|\mathbf{b}_{0}\right\|\right) \mathbf{E}_{t} \widetilde{\mathbf{a}}\right\|^{2}-2 \mathbb{E} \mathbf{e}_{t}^{\prime}\left(\mathbf{B}^{\prime} /\left\|\mathbf{b}_{0}\right\|\right) \mathbf{E}_{t} \widetilde{\mathbf{a}}
$$

Now $\mathbf{E}_{t} \widetilde{\mathbf{a}}=\left(\left(\mathbf{e}_{t}^{\prime} \mathbf{b}_{0}\right) /\left\|\mathbf{b}_{0}\right\|, \ldots,\left(\mathbf{e}_{t-k_{2}}^{\prime} \mathbf{b}_{0}\right) /\left\|\mathbf{b}_{0}\right\|\right)^{\prime}$ and hence

$$
\left\|\mathbf{E}_{t} \widetilde{\mathbf{a}}\right\|^{2}=\sum_{h=0}^{k_{2}}\left(\mathbf{e}_{t-h}^{\prime} \mathbf{b}_{0}\right)^{2} /\left\|\mathbf{b}_{0}\right\|^{2}
$$

It follows that $\mathbb{E}\left\|\mathbf{E}_{t} \widetilde{\mathbf{a}}\right\|^{2}=\left(k_{2}+1\right)\left(\left(1 /\left\|\mathbf{b}_{0}\right\|^{2}\right) \mathbf{b}_{0}^{\prime} \boldsymbol{\Sigma}^{e}(0) \mathbf{b}_{0}\right)$. Hence, using Condition $\left.2 \mathrm{a}\right)$ and c)

$$
\frac{1}{m} \mathbb{E}\left\|\left(\mathbf{B}^{\prime} /\left\|\mathbf{b}_{0}\right\|\right) \mathbf{E}_{t} \widetilde{\mathbf{a}}\right\|^{2} \leq \frac{\left\|\mathbf{B}^{\prime}\right\|^{2}}{m}\left(k_{2}+1\right)\left(\left(1 /\left\|\mathbf{b}_{0}\right\|^{4}\right) \mathbf{b}_{0}^{\prime} \boldsymbol{\Sigma}^{e}(0) \mathbf{b}_{0}\right)=o(1) .
$$

Finally,

$$
\frac{1}{m\left\|\mathbf{b}_{0}\right\|} \mathbb{E}\left|\mathbf{e}_{t}^{\prime} \mathbf{B}^{\prime} \mathbf{E}_{t} \widetilde{\mathbf{a}}\right| \leq\left(\mathbb{E}\left\|\mathbf{e}_{t}\right\|^{2} / m\right)^{1 / 2}\left(\mathbb{E}\left\|\left(\mathbf{B}^{\prime} /\left\|\mathbf{b}_{0}\right\|\right) \mathbf{E}_{t} \widetilde{\mathbf{a}}\right\|^{2} / m\right)^{1 / 2}=o(1) .
$$

Since $\mathbb{E}\left\|\mathbf{e}_{t}\right\|^{2}=\sum_{j=1}^{m} \mathbb{E} e_{t, j}^{2}$, the Theorem is proven. 
We will need the following general results on linear predictors.

Lemma 6. Let $X$ and $Z_{1}, \ldots, Z_{n}$ be zero mean random variables satisfying $\mathbb{E} Z_{i}^{2} \leq b$, for some $b>0$ and $i=1, \ldots, n$, and $\mathbb{E} Z_{i} Z_{j}=0$ for $i \neq j$. Let $P\left(X \mid Z_{i}\right)$ be the best linear predictor of $X$ based on $Z_{i}$. Then

$$
\sum_{i=1}^{n} \mathbb{E} P\left(Z_{i} \mid X\right)^{2} \leq b
$$

Proof of Lemma 6 . Let $P(X \mid Z)$ be the best linear predictor of $X$ based on $Z_{1}, \ldots Z_{n}$. Since $Z_{1}, \ldots, Z_{n}$ are uncorrelated, we have

$$
P(X \mid Z)=\sum_{i=1}^{n} P\left(X \mid Z_{i}\right)=\sum_{i=1}^{n} \frac{\mathbb{E} X Z_{i}}{\mathbb{E} Z_{i}^{2}} Z_{i}
$$

Hence

$$
\mathbb{E} X^{2} \geq \mathbb{E} P(X \mid Z)^{2}=\sum_{i=1}^{n} \mathbb{E} P\left(X \mid Z_{i}\right)^{2}=\sum_{i=1}^{n} \frac{\left(\mathbb{E} X Z_{i}\right)^{2}}{\mathbb{E} Z_{i}^{2}} \geq(1 / b) \sum_{i=1}^{n}\left(\mathbb{E} X Z_{i}\right)^{2}
$$

Now,

$$
\sum_{i=1}^{n} \mathbb{E} P\left(Z_{i} \mid X\right)^{2}=\sum_{i=1}^{n} \mathbb{E}\left(\frac{\mathbb{E} X Z_{i}}{\mathbb{E} X^{2}} X\right)^{2}=\sum_{i=1}^{n} \frac{\left(\mathbb{E} X Z_{i}\right)^{2}}{\mathbb{E} X^{2}} \leq b
$$

Lemma 7. Let $Z_{1}, \ldots, Z_{n}$ be zero mean random variables satisfying $\mathbb{E} Z_{i}^{2} \leq b$, for some $b>0$ and $i=1, \ldots, n$, and $\mathbb{E} Z_{i} Z_{j}=0$ for $i \neq j$. Let $Y_{1}, \ldots, Y_{k}$ be zero mean random variables and $P\left(Z_{i} \mid Y\right)$ be the best linear predictor of $Z_{i}$ based on $Y_{1}, \ldots, Y_{k}$. Then

$$
\liminf _{n \rightarrow \infty} \frac{1}{n} \sum_{i=1}^{n}\left(\mathbb{E}\left(Z_{i}-P\left(Z_{i} \mid Y\right)\right)^{2}-\mathbb{E} Z_{i}^{2}\right) \geq 0 .
$$


Proof of Lemma 4. Let $X_{1}, \ldots, X_{k}$ be zero mean, unit variance, uncorrelated random variables with the same linear span as $Y_{1}, \ldots, Y_{k}$. Let $P\left(Z_{i} \mid X\right)$ be the best linear predictor of $Z_{i}$ based on $X_{1}, \ldots, X_{k}$. Then, using Lemma 6

$$
\begin{aligned}
\sum_{i=1}^{n} \mathbb{E}\left(Z_{i}-P\left(Z_{i} \mid Y\right)\right)^{2} & =\sum_{i=1}^{n} \mathbb{E}\left(Z_{i}-P\left(Z_{i} \mid X\right)\right)^{2}=\sum_{i=1}^{n}\left(\mathbb{E} Z_{i}^{2}-\mathbb{E} P\left(Z_{i} \mid X\right)^{2}\right) \\
& =\sum_{i=1}^{n} \mathbb{E} Z_{i}^{2}-\sum_{i=1}^{n} \sum_{j=1}^{k} \mathbb{E} P\left(Z_{i} \mid X_{j}\right)^{2} \geq \sum_{i=1}^{n} \mathbb{E} Z_{i}^{2}-k b .
\end{aligned}
$$

Hence

$$
\liminf _{n \rightarrow \infty} \frac{1}{n} \sum_{i=1}^{n}\left(\mathbb{E}\left(Z_{i}-P\left(Z_{i} \mid Y\right)\right)^{2}-\mathbb{E} Z_{i}^{2}\right) \geq \lim _{n \rightarrow \infty}-\frac{k b}{n}=0
$$

Proof of Theorem 3 .

$$
\begin{aligned}
\frac{1}{m} \operatorname{MSE}_{0}\left(\mathbf{a}^{*}, \mathbf{B}^{*}\right) & =\frac{1}{m} \mathbb{E}\left\|\mathbf{z}_{t}-\widehat{\mathbf{z}}_{t}\right\|^{2}=\frac{1}{m} \mathbb{E}\left\|\mathbf{B}^{\prime} \mathbf{f}_{t}-\widehat{\mathbf{z}}_{t}+\mathbf{e}_{t}\right\|^{2} \\
& =\frac{1}{m} \mathbb{E}\left\|\mathbf{B}^{\prime} \mathbf{f}_{t}-\widehat{\mathbf{z}}_{t}\right\|^{2}+\frac{1}{m} \mathbb{E}\left\|\mathbf{e}_{t}\right\|^{2}+2 \mathbb{E} \mathbf{e}_{t}^{\prime}\left(\mathbf{B}^{\prime} \mathbf{f}_{t}-\widehat{\mathbf{z}}_{t}\right) .
\end{aligned}
$$

By Condition 2r)

$$
\mathbb{E} \mathbf{e}_{t}^{\prime}\left(\mathbf{B}^{\prime} \mathbf{f}_{t}-\widehat{\mathbf{z}}_{t}\right)=\mathbb{E} \mathbf{e}_{t}^{\prime} \widehat{\mathbf{z}}_{t}
$$

By Theorem 2, it suffices to show that

$$
\frac{1}{m} \mathbb{E} \mathbf{e}_{t}^{\prime} \widehat{\mathbf{z}}_{t} \rightarrow 0
$$

Let $\widehat{e}_{t, j}$ be the best linear predictor of $e_{t, j}$ based on $\widehat{z}_{t, j}$. Then

$$
\mathbb{E}\left(e_{t, j}-\widehat{e}_{t, j}\right)^{2}=\mathbb{E} e_{t, j}^{2}-\frac{\left(\mathbb{E} \widehat{z}_{t, j} e_{t, j}\right)^{2}}{\mathbb{E} \widehat{z}_{t, j}^{2}}
$$


Note that $\mathbb{E} \widehat{z}_{t, j}^{2} \leq \mathbb{E} z_{t, j}^{2} \leq L$, since $\widehat{z}_{t, j}$ is obtained by projecting $z_{t, j}$ on the space spanned by $\widehat{\mathbf{f}}_{t}$. Then

$$
\frac{1}{m} \sum_{j=1}^{m} \mathbb{E}\left(e_{t, j}-\widehat{e}_{t, j}\right)^{2} \leq \frac{1}{m} \sum_{j=1}^{m}\left(\mathbb{E} e_{t, j}^{2}-\frac{\left(\mathbb{E} \widehat{z}_{t, j} e_{t, j}\right)^{2}}{L}\right) .
$$

Since by Lemma 7, $\lim \inf (1 / m) \sum_{j=1}^{m}\left(\mathbb{E}\left(e_{t, j}-\widehat{e}_{t, j}\right)^{2}-\mathbb{E} e_{t, j}^{2}\right) \geq 0$ it must be that

$$
(1 / m) \sum_{j=1}^{m}\left(\mathbb{E} \widehat{z}_{t, j} e_{t, j}\right)^{2} \rightarrow 0,
$$

from which the result follows by applying the Cauchy-Schwartz inequality.

\section{References}

Adams, T. M. and Nobel, A. B. (2010). Uniform convergence of Vapnik-Chervonenkis classes under ergodic sampling. Ann. Probab., 38(4):1345-1367.

Ahn, S. K. and Reinsel, G. C. (1988). Nested reduced-rank autogressive models for multiple time series. Journal of the American Statistical Association, 83(403):849-856.

Bai, J. and Ng, S. (2002). Determining the number of factors in approximate factor models. Econometrica, 70(1):191-221.

Box, G. E. P. and Tiao, G. C. (1977). A canonical analysis of multiple time series. Biometrika, 64(2):355.

Brillinger, D. R. (1964). The generalization of the techniques of factor analysis, canonical correlation and principal components to stationary time series. Invited Paper at the Royal Statistical Society Conference in Cardiff, Wales. 
Brillinger, D. R. (1981). Time Series: Data Analysis and Theory. Classics in Applied Mathematics. Society for Industrial and Applied Mathematics.

Connor, G. and Korajczyk, R. A. (1993). A test for the number of factors in an approximate factor model. The Journal of Finance, 48(4):1263-1291.

Forni, M., Hallin, M., Lippi, M., and Reichlin, L. (2000). The generalized dynamic-factor model: Identification and estimation. The Review of Economics and Statistics, 82(4):540554.

Forni, M., Hallin, M., Lippi, M., and Reichlin, L. (2005). The generalized dynamic factor model: One-sided estimation and forecasting. Journal of the American Statistical Association, 100(471):830-840.

Forni, M., Hallin, M., Lippi, M., and Zaffaroni, P. (2015a). Dynamic factor models with infinite-dimensional factor spaces: Asymptotic analysis. Technical report, ECARES.

Forni, M., Hallin, M., Lippi, M., and Zaffaroni, P. (2015b). Dynamic factor models with infinite-dimensional factor spaces: One-sided representations. Journal of Econometrics, $185(2): 359-371$.

Garcia-Ferrer, A., Highfield, R. A., Palm, F., and Zellner, A. (1987). Macroeconomic forecasting using pooled international data. Journal of Business $\&$ Economic Statistics, $5(1): 53-67$.

Horn, R. and Johnson, C. (1994). Topics in Matrix Analysis. Cambridge University Press.

Hyndman, R. and Khandakar, Y. (2008). Automatic time series forecasting: The forecast package for R. Journal of Statistical Software, 27(1):1-22. 
Litterman, R. B. (1986). Forecasting with bayesian vector autoregressions: Five years of experience. Journal of Business 86 Economic Statistics, 4(1):25-38.

McCracken, M. W. and Ng, S. (2016). Fred-md: A monthly database for macroeconomic research. Journal of Business 85 Economic Statistics, 4(34):574-589.

Peña, D. and Box, G. E. P. (1987). Identifying a simplifying structure in time series. Journal of the American Statistical Association, 82(399):836-843.

Peña, D. and Poncela, P. (2004). Forecasting with nonstationary dynamic factor models. Journal of Econometrics, 119(2):291-321.

Peña, D. and Yohai, V. J. (2016). Generalized dynamic principal components. Journal of the American Statistical Association, 111(515):1121-1131.

Smucler, E. and Yohai, V. J. (2017). Robust and sparse estimators for linear regression models. Computational Statistics $\&$ Data Analysis, 111(C):116-130.

Stock, J. H. and Watson, M. W. (2002). Forecasting using principal components from a large number of predictors. Journal of the American Statistical Association, 97(460):11671179.

Tiao, G. C. and Tsay, R. S. (1989). Model specification in multivariate time series. Journal of the Royal Statistical Society. Series B (Methodological), 51(2):157-213.

Yohai, V. J. (1987). High breakdown-point and high efficiency robust estimates for regression. The Annals of Statistics, 15(2):642-656. 